\title{
Outflow, Infall, and Protostars in the Star-forming Core W3-se
}

\section{Citation}

Zhu, Lei, Jun-Hui Zhao, and M. C. H. Wright. 2011. “Outflow, Infall, and Protostars

in the Star-forming Core W3-se." The Astrophysical Journal 740 (2): 114. https://

doi.org/10.1088/0004-637x/740/2/114.

\section{Permanent link}

http://nrs.harvard.edu/urn-3:HUL.InstRepos:41555824

\section{Terms of Use}

This article was downloaded from Harvard University's DASH repository, and is made available under the terms and conditions applicable to Other Posted Material, as set forth at http:// nrs.harvard.edu/urn-3:HUL.InstRepos:dash.current.terms-of-use\#LAA

\section{Share Your Story}

The Harvard community has made this article openly available.

Please share how this access benefits you. Submit a story.

Accessibility 


\title{
OUTFLOW, INFALL, AND PROTOSTARS IN THE STAR-FORMING CORE W3-SE
}

\author{
Lei ZhU ${ }^{1,2}$, Jun-Hui ZhaO ${ }^{2}$, AND M. C. H. Wright ${ }^{3}$ \\ ${ }^{1}$ Department of Astronomy, Peking University, Beijing 100871, China \\ ${ }^{2}$ Harvard-Smithsonian Center for Astrophysics, 60 Garden Street, Cambridge, MA 02138, USA; lzhu@ cfa.harvard.edu, jzhao@cfa.harvard.edu \\ ${ }^{3}$ Department of Astronomy, University of California Berkeley, Berkeley, CA 94720, USA \\ Received 2010 December 17; accepted 2011 July 22; published 2011 October 4
}

\begin{abstract}
We report new results on outflow and infall in the star-forming cores W3-SE SMA-1 and SMA-2 based on analysis of $\sim 2$ '. 5 resolution observations of the molecular lines $\mathrm{HCN}(3-2), \mathrm{HCO}^{+}(3-2), \mathrm{N}_{2} \mathrm{H}^{+}(3-2)$, and $\mathrm{CH}_{3} \mathrm{OH}\left(5_{2,3}-4{ }_{1,3}\right)$ with the Submillimeter Array (SMA). A high-velocity bipolar outflow originating from the protostellar core SMA-1 was observed in the $\mathrm{HCN}(3-2)$ line, with a projected outflow axis at a position angle of $48^{\circ}$. The detection of the outflow is confirmed from other molecular lines. An inverse P-Cygni profile in the HCN(3-2) line toward SMA-1 suggests that at least one of the double cores accretes matter from the molecular core. A filamentary structure in the molecular gas surrounds SMA-1 and SMA-2. Based on the SMA observations, our analysis suggests that the double pre-stellar cores SMA-1 and SMA-2 result from fragmentation in the collapsing massive molecular core W3-SE, and it is likely that they are forming intermediate- to high-mass stars which will be new members of a star cluster in the W3-SE region.
\end{abstract}

Key words: ISM: clouds - ISM: jets and outflows - ISM: kinematics and dynamics - ISM: molecules - radio lines: ISM

Online-only material: color figures

\section{INTRODUCTION}

One of the key questions in the study of star formation is how protostars accrete material from their parent molecular clouds. Accretion around protostars with intermediate and high mass is not well understood due to the lack of high-resolution observations. Observation of infall is needed to provide direct evidence for accretion. Low-resolution observations of a bluedominated double-peaked spectral profile as expected from a model of self-absorption in infalling gas clumps (NGC 1333IRAS 2 as an example; see Ward-Thompson et al. 1996; WardThompson \& Buckley 2001; Jorgensen et al. 2004a; Peretto et al. 2006) do not unambiguously identify infall because massive cores usually contain bipolar outflows, disk rotation, and multiple sub-cores in addition to gas infall. Complex dynamical processes can produce characteristics similar to those expected from the self-absorption model of a molecular core with infall. It is crucial to carry out high angular and spectral resolution observations along with comprehensive modeling of the dynamical processes involved in a massive star-forming region.

There is growing evidence that massive stars form in groups, but accretion modeling has been for material concentrated in monolithic cores (McKee \& Ostriker 2007). The formation of binary and multiple stellar systems, especially those with two comparable-mass protostars and a large separation, is poorly understood (McKee \& Ostriker 2007). Detailed highresolution observations of a binary/multiple protostellar system will provide valuable data for understanding accretion and outflow from each of the sub-cores in the system.

W3-SE is a dense molecular core located SE of the wellknown massive star formation region W3-Main. We adopted a distance of $D=2 \mathrm{kpc}$ for W3-SE (Blitz et al. 1982; Xu et al. 2006). The spectral energy distribution (SED), along with a double continuum core revealed in our previous paper ( $\mathrm{Zhu}$ et al. 2010), suggests that a total mass $65 M_{\odot}$ for the W3-SE core over a scale of $10^{\prime \prime}$ is mainly composed of two protostellar cores of comparable masses, which are separated by $3 . .5$ or $7000 \mathrm{AU}$ as revealed in the continuum observations with the Submillimeter Array (SMA). ${ }^{4}$ Several mid-IR point sources are identified in the W3-SE region with Spitzer IRAC data (Ruch et al. 2007; Zhu et al. 2010). Low resolution observations with a single-dish telescope show a blue profile, a possible infall signature, toward W3-SE (Wu et al. 2007). Observations of $\mathrm{HCO}^{+}(1-0)$ using the CARMA telescope at $6^{\prime \prime}$ resolution show that outflows appear to dominate the observed spectra although infall might also contribute to the spectrum toward the main molecular core which coincides with the double cores. Spectral line observations with higher angular resolution are necessary to separate the contributions of infall and outflow from the double cores in W3-SE and to investigate the possibility of a protobinary system.

In this paper, we present results of an analysis of spectral line data observed with the SMA at $\sim 2$ ". 5 resolution. Section 2 describes the observations and data reductions. Section 3 shows the results on the basis of our analysis of the molecular line data including $\mathrm{HCN}(3-2), \mathrm{HCO}^{+}(3-2), \mathrm{N}_{2} \mathrm{H}^{+}(3-2)$, and $\mathrm{CH}_{3} \mathrm{OH}\left(5_{2,3}-4_{1,3}\right)$. In Section 4, we present an interpretation of the observations in the context of outflow and infall. Section 5 gives a summary and conclusions.

\section{OBSERVATIONS}

The observations toward W3-SE consist of two tracks obtained at $\lambda 1.1 \mathrm{~mm}$ using the SMA in the compact configuration with an angular resolution of $\sim 2$ 2.5 in 2008 October 27 and December 20. The observations were centered at R.A.(J2000) $=$ $02^{\mathrm{h}} 25^{\mathrm{m}} 54.50$, decl. $(\mathrm{J} 2000)=62^{\circ} 04^{\prime} 11^{\prime \prime} .0$, which is used as the reference position in the following analysis. The local oscillator (LO) frequency $\left(v_{\mathrm{LO}}\right)$ for the 2008 October track was tuned to

\footnotetext{
4 The Submillimeter Array is a joint project between the Smithsonian Astrophysical Observatory and the Academia Sinica Institute of Astronomy and Astrophysics and is funded by the Smithsonian Institution and the Academia Sinica.
} 

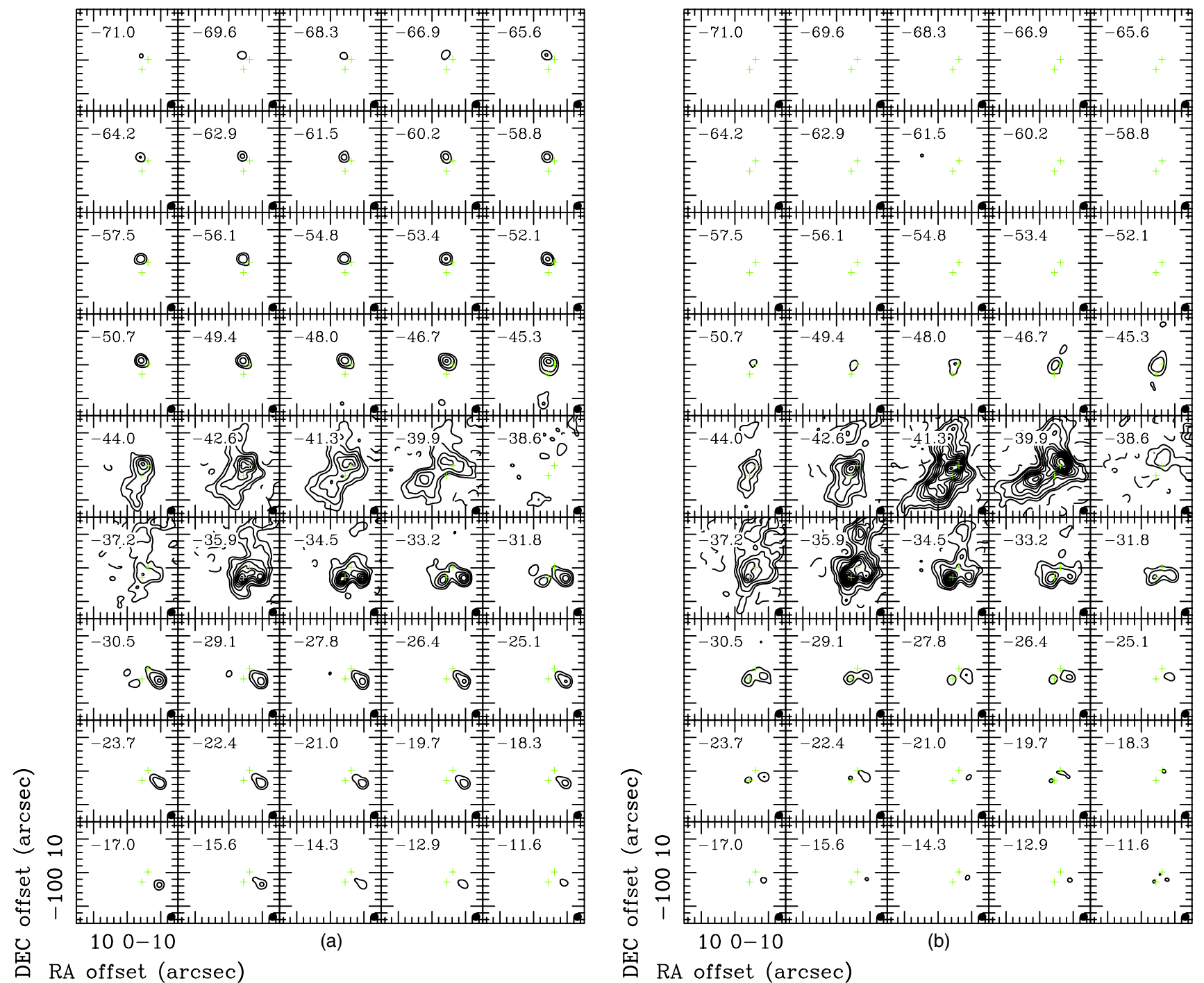

Figure 1. Channel maps of $\mathrm{HCN}(3-2)$ (a: left panel) and $\mathrm{HCO}^{+}(3-2)$ (b: right panel) with a velocity interval of $1.4 \mathrm{~km} \mathrm{~s}^{-1}$. Contours are $(-1,1,2,4,6, \ldots) \times 5 \sigma, \sigma$ $=0.045 \mathrm{Jy}_{\text {beam }}^{-1}$ in both maps with an FWHM beam of $2^{\prime \prime} .5 \times 2.3\left(-65^{\circ}\right)$ as marked at right bottom. The number at left top in each channel image corresponds to the radial velocity. The green crosses mark the positions of the two protostellar objects, SMA-1 and SMA-2.

(A color version of this figure is available in the online journal.)

$271.7 \mathrm{GHz}$ and $v_{\mathrm{LO}}=285.3 \mathrm{GHz}$ for the other. The observations consist of a lower sideband (LSB) and an upper sideband (USB) separated by $10 \mathrm{GHz}$, with a bandwidth of $2 \mathrm{GHz}$ for each sideband. The typical system temperatures $\left(T_{\text {sys }}\right)$ are $180-200 \mathrm{~K}$ and $200-250 \mathrm{~K}$ for the observations in 2008 October and December, respectively.

Data reduction and imaging were carried out in MIRIAD (Sault et al. 1995) with specific implementations for SMA data reduction. ${ }^{5}$ The bandpass was calibrated using QSOs $3 \mathrm{C} 454.3$ for the lower-frequency track and 3C273 for the higher-frequency track. For both tracks the complex gains were calibrated using QSO 0244+624. The flux density scale was bootstrapped from Uranus using the SMA planet model. By combining the visibility data from both LSB and USB, the continuum images achieve rms noises of $1.5 \mathrm{mJy}$ and $3.0 \mathrm{mJy}$ for the data observed in 2008 October and December, respectively. Spectral line images were made by subtracting the continuum emission from each set of visibility data using the MIRIAD task UVLIN.

\footnotetext{
5 http://www.cfa.harvard.edu/sma/miriad
}

\section{RESULTS}

\section{1. $\mathrm{HCN}(3-2)$}

A transition of hydrogen cyanide, $\operatorname{HCN}(3-2)$ at $v_{0}=$ $265.886 \mathrm{GHz}$, was observed in the LSB of the 2008 October observations. Figure 1(a) presents the $\mathrm{HCN}(3-2)$ channel maps, showing multiple velocity components distributed in the W3-SE core. In the velocity range between -39.3 and $-38.0 \mathrm{~km} \mathrm{~s}^{-1}$ a gap with no significant line emission signals is observed. The figure also shows compact $\mathrm{HCN}(3-2)$ components in the high-velocity channels $\left(<-46.7 \mathrm{~km} \mathrm{~s}^{-1}\right.$ and $>-30.5 \mathrm{~km} \mathrm{~s}^{-1}$ ). Figure 2(a) shows the integrated line intensity map of $\mathrm{HCN}(3-2)$, suggesting the presence of three emission peaks from the $\mathrm{HCN}(3-2)$ line within the main molecular emission component $\mathrm{A}$, as observed by CARMA at an angular resolution of 6" (Zhu et al. 2010). We denote the three sub-components as A-NE, A-SW, and A-SE, respectively. The $\mathrm{HCN}(3-2)$ line components A-NE and A-SW are located 2" northeast and $4 "$ southwest of the protostellar core SMA-1 while the component A-SE nearly coincides with SMA-2. The integrated intensity of 

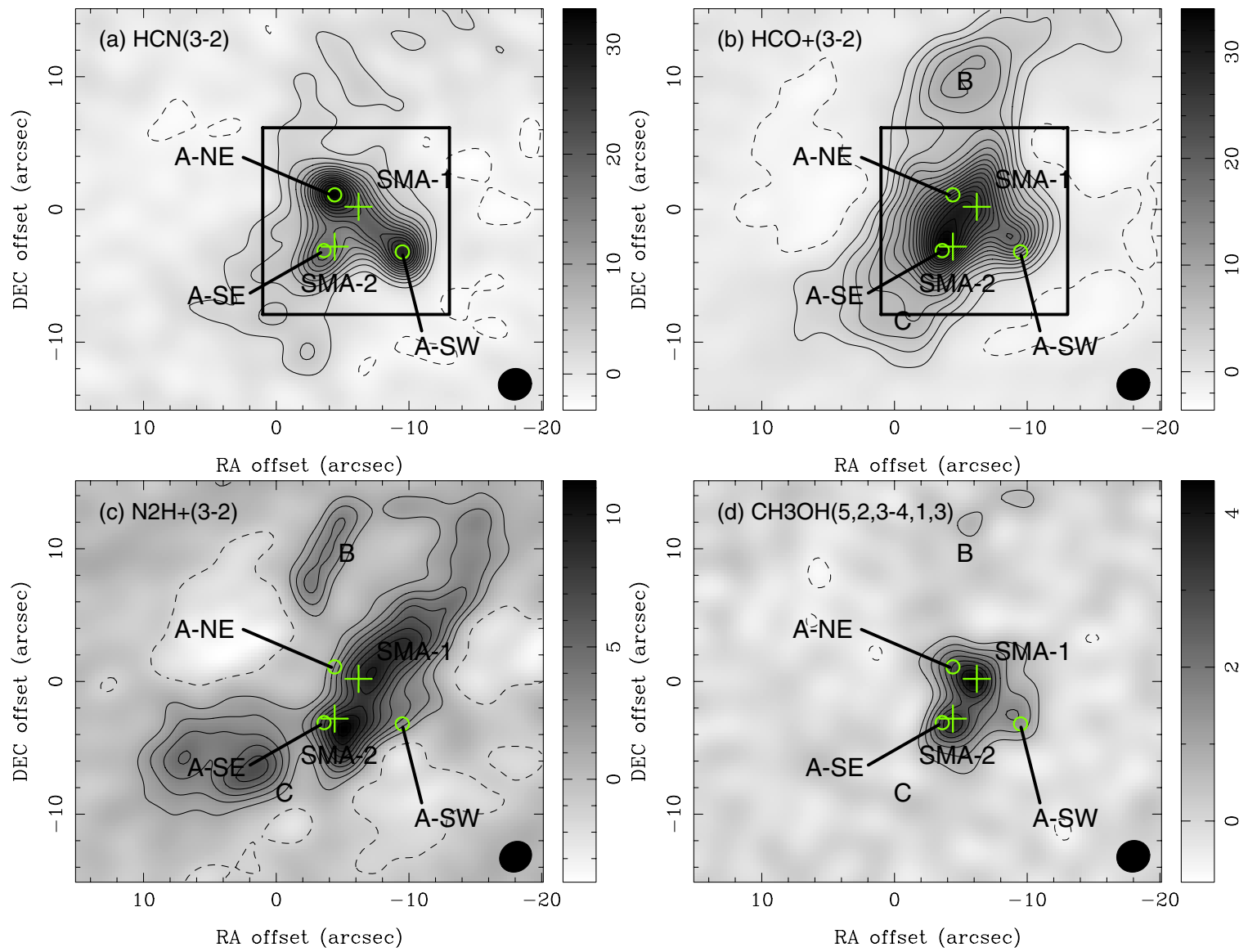

Figure 2. Integrated intensity maps of (a) $\mathrm{HCN}(3-2)$ in the velocity range of $(-70.5,-9.8) \mathrm{km} \mathrm{s}^{-1}$, (b) $\mathrm{HCO}^{+}(3-2)$ in $(-53.0,-21.0) \mathrm{km} \mathrm{s}^{-1}$, (c) $\mathrm{N}_{2} \mathrm{H}^{+}(3-2)$ in $(-43.5,-34.5) \mathrm{km} \mathrm{s}^{-1}$, and (d) $\mathrm{CH}_{3} \mathrm{OH}\left(5_{2,3}-4,3\right)$ in $(-44.5,-33.7) \mathrm{km} \mathrm{s}^{-1}$. Contours are $(-1,1,2,3,4 \ldots) \times 3 \sigma \mathrm{Jy} \mathrm{beam}^{-1} \mathrm{~km} \mathrm{~s}^{-1}$. The rms noises $(1 \sigma)$ are $\sim 0.7$, $\sim 0.7, \sim 0.5$, and $\sim 0.2 \mathrm{Jy} \mathrm{beam}^{-1} \mathrm{~km} \mathrm{~s}^{-1}$ for the images (a), (b), (c), and (d), respectively. The images (a), (b), and (d) were made from the LSB data observed at $v_{\mathrm{LO}}=271.7 \mathrm{GHz}$ with a FWHM beam of 2". $5 \times 2$ '. $3\left(-65^{\circ}\right)$. The FWHM beam of the image (c) (USB data) is $2^{\prime \prime} .5 \times 2^{\prime \prime} \cdot 2\left(-55^{\circ}\right)$. Crosses denote the positions of the protostellar objects SMA-1 and SMA-2. Open circles mark the three peak positions of the HCN(3-2) line emission (A-NE, A-SW, and A-SE). The positions of the weaker emission components $\mathrm{B}$ and $\mathrm{C}$ observed in $\mathrm{HCO}^{+}(1-0)$ with CARMA are also indicated.

(A color version of this figure is available in the online journal.)

$\mathrm{HCN}(3-2)$ line emission in A-NE and A-SW is stronger than that from A-SE.

Figure 3 shows the spectra of the $\mathrm{HCN}(3-2)$ line in the core region of W3-SE with a boundary outlined by the square in Figure 2(a). A significant absorption feature is shown in the core region at velocities in the range between -39.3 and $-38.0 \mathrm{~km} \mathrm{~s}^{-1}$. The component A-NE appears to be associated with a broad blueshifted line wing, while A-SW shows a broad redshifted line wing. The highly blueshifted line wing associated with A-NE shows a maximum absolute radial velocity of $35 \mathrm{~km} \mathrm{~s}^{-1}$ (above the $5 \sigma$ cutoff), with respect to SMA-1. Here a local standard of rest (LSR) systemic velocity $V_{\text {sys }}=-39.1 \mathrm{~km} \mathrm{~s}^{-1}$ is used, which is determined from the peak velocity of the optically thin line $\mathrm{CH}_{3} \mathrm{OH}\left(5_{2,3}-4_{1,3}\right)$. In the region of A-SW, the redshifted line wing shows a maximum absolute radial velocity of $33 \mathrm{~km} \mathrm{~s}^{-1}$ (above $5 \sigma$ ) with respect to SMA-1. The velocity profiles for both blueshifted and redshifted broad-line wings can be fitted to a power law $\left(\sim\left|V-V_{\text {sys }}\right|^{-\gamma}\right)$ with an index of $\gamma \approx 2.0$. The power-law distribution of molecular content as function of velocity observed is consistent with numerical models of bow-shock outflow (e.g., Downes \& Ray 1999; Lee et al. 2001).

In Figure 4(a), we show the high-velocity gas in both the blueshifted $\left(-74.1\right.$ to $\left.-45.2 \mathrm{~km} \mathrm{~s}^{-1}\right)$ and redshifted $(-31.9$ to $-5.8 \mathrm{~km} \mathrm{~s}^{-1}$ ) outflow lobes, located NE and $\mathrm{SW}$ of the protostellar core SMA-1 with separations of 2.2 and 5.0 , respectively. The configuration of the emission from the highvelocity wings, along with the location of SMA-1, suggests a bipolar outflow originating from SMA-1, a possible energy source in the region. The major axis of the outflow is shown as a straight line connecting the two peaks of the blueshifted and redshifted knots in Figure 4(a), at a position angle (P.A.) of $48^{\circ}$. Although the location of SMA-1 appears to be offset from the outflow axis by $\sim 0^{\prime} 4$, about $5 \%$ of the distance between the two high-velocity knots, such an apparent offset could be due to the uncertainty $\left(\sim 0^{\prime} ! 2\right)$ in the peak position of SMA-1 given by Gaussian fitting.

In summary, the high-velocity $\mathrm{HCN}(3-2)$ line emission from the two compact molecular components A-NE and A-SW appears to be excited by a high-velocity bipolar outflow originating from the protostellar core SMA-1.

\section{2. $\mathrm{HCO}^{+}(3-2)$}

The transition of $\mathrm{HCO}^{+}(3-2)$ at $267.558 \mathrm{GHz}$ was also included in the LSB of the observations at $v_{\mathrm{LO}}=271.7 \mathrm{GHz}$. Figure 1(b) shows the channel maps of the $\mathrm{HCO}^{+}(3-2)$ line. The overall distribution of $\mathrm{HCO}^{+}$gas in velocity is consistent with that of $\mathrm{HCO}^{+}(1-0)$ observed with CARMA (Zhu et al. 2010) and similar to that of $\mathrm{HCN}(3-2)$ in the SMA observations, showing 


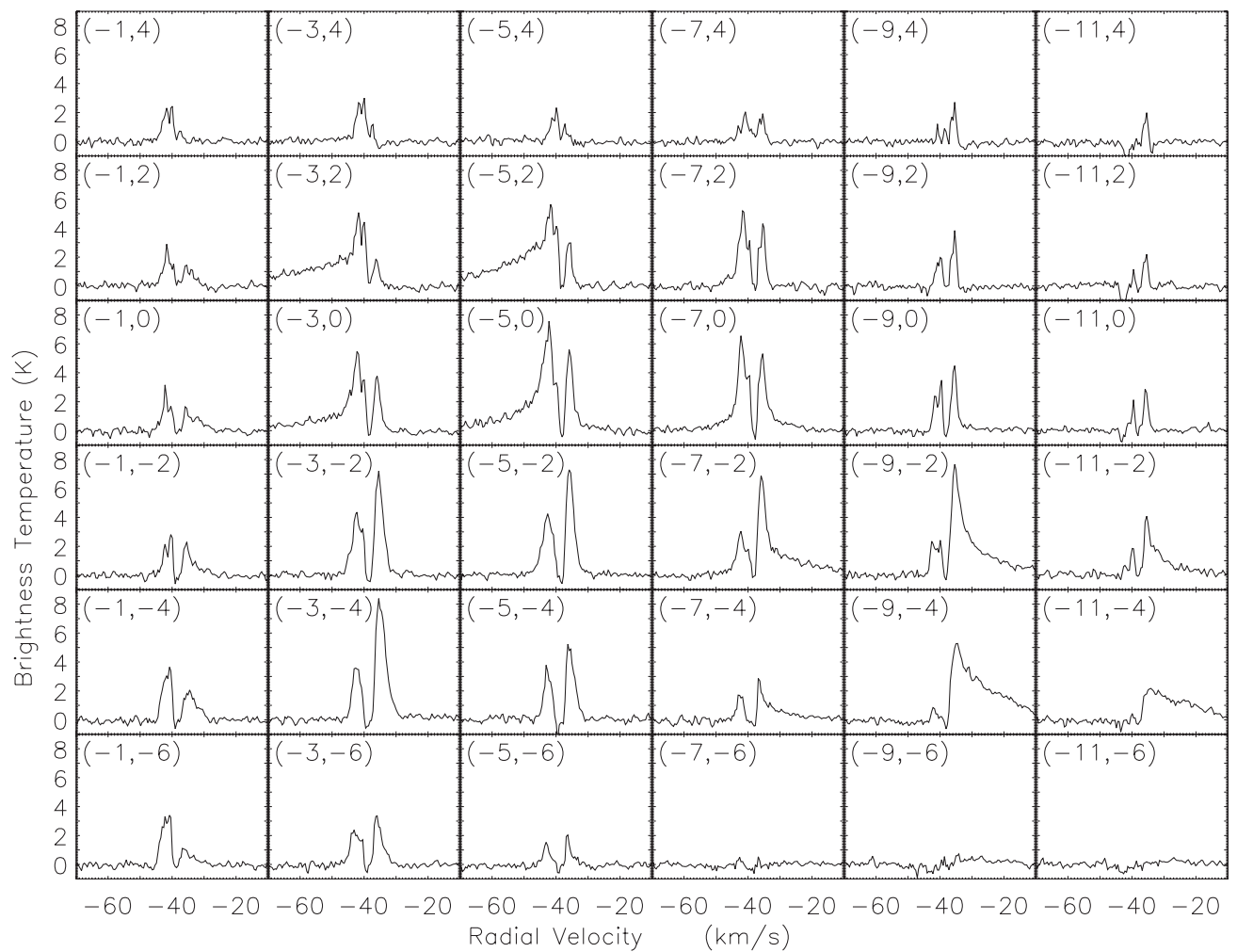

Figure 3. Grid of spectra for the $\mathrm{HCN}(3-2)$ line emission. The region covered is marked as the black rectangle in Figure 2(a). The pair of numbers in parentheses at top left in each of the spectra gives the offset in units of arcseconds with respect to the reference center.
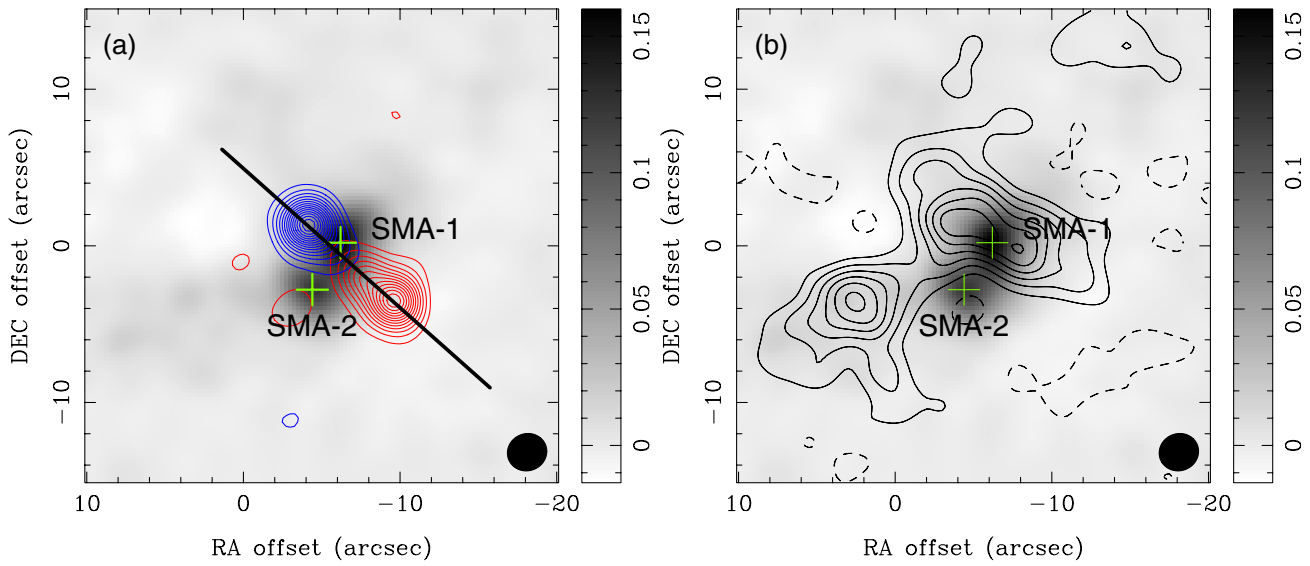

Figure 4. (a) The high-velocity outflow map in $\mathrm{HCN}(3-2)$, integrated from the blue wing in the velocity range $(-74.1,-45.2) \mathrm{km} \mathrm{s}^{-1}$ (blue contours) and the red wing in $(-31.9,-5.8) \mathrm{km} \mathrm{s}^{-1}$ (red contours). Contours are $(-1,1,2,3,4 \ldots) \times 5 \sigma ; 1 \sigma=0.28 \mathrm{Jy} \mathrm{beam}{ }^{-1} \mathrm{~km} \mathrm{~s}^{-1}$. The position angle of the outflow axis (the thick straight line) is $48^{\circ}$. (b) The integrated intensity map of the blueshifted $\mathrm{HCN}(3-2)$ emission bump in the velocity range of $(-39.9,-39.5) \mathrm{km} \mathrm{s}^{-1}$, which could somehow trace the distribution of possible infalling gas. Contours are $(-1,3,2,3,4 \ldots) \times 3 \sigma ; 1 \sigma=0.1 \mathrm{Jy} \mathrm{beam}^{-1} \mathrm{~km} \mathrm{~s}^{-1}$. For both the figures the FWHM beam is $2^{\prime \prime} .5 \times 2^{\prime \prime} .3$, P.A. $=$ $-65^{\circ}$. For both figures the background shows the $1.1 \mathrm{~mm}$ continuum distribution, and the wedges are in units of Jy beam ${ }^{-1}$.

multiple emission peaks at various velocities and a gap in the velocity range between -38.9 and $-37.9 \mathrm{~km} \mathrm{~s}^{-1}$.

However, not all of the emission peaks observed in $\mathrm{HCO}^{+}(3-2)$ coincide with those in $\mathrm{HCN}(3-2)$. In the integrated intensity map of $\mathrm{HCO}^{+}(3-2)$ in Figure 2(b), the emission peak A-NE observed in $\mathrm{HCN}(3-2)$ appears to be embedded in the surrounding emission, and $\mathrm{A}-\mathrm{SW}$ in $\mathrm{HCO}^{+}(3-2)$ is not as prominent as it is in $\mathrm{HCN}(3-2)$. Instead, strong emission in $\mathrm{HCO}^{+}(3-2)$ appears to be concentrated in a bright ridge 0.5 northeast of the line connecting the double protostellar cores SMA-1 and SMA2. Outside the main core A, the molecular sub-core B and the molecular tail $\mathrm{C}$ are also present, in good agreement with the image of $\mathrm{HCO}^{+}(1-0)$ observed with CARMA. In comparison with the $\mathrm{HCN}(3-2)$ image, the $\mathrm{HCO}^{+}(3-2)$ line appears to trace a more extended envelope around the double protostellar cores.

As Figure 5 shows, the observed $\mathrm{HCO}^{+}(3-2)$ spectra are also found with broad-line wings at the position of SMA-1 and its adjacent region, although the line wings in $\mathrm{HCO}^{+}(3-2)$ are not as significant and broad as those in the $\mathrm{HCN}(3-2)$ spectra. The integrated intensity map for the blueshifted and redshifted line wings is shown in Figure 6(a). The velocity ranges for the integrations are $(-57.9,-45.3) \mathrm{km} \mathrm{s}^{-1}$ and $(-31.8,-18.8) \mathrm{km} \mathrm{s}^{-1}$, with the same lower velocity limits as for $\mathrm{HCN}(3-2)$. In Figure 6(a), the compact blueshifted and redshifted lobes are offset from SMA-1 similar to the case of $\mathrm{HCN}(3-2)$, although they are closer to SMA-1 (1".3 for the blue 


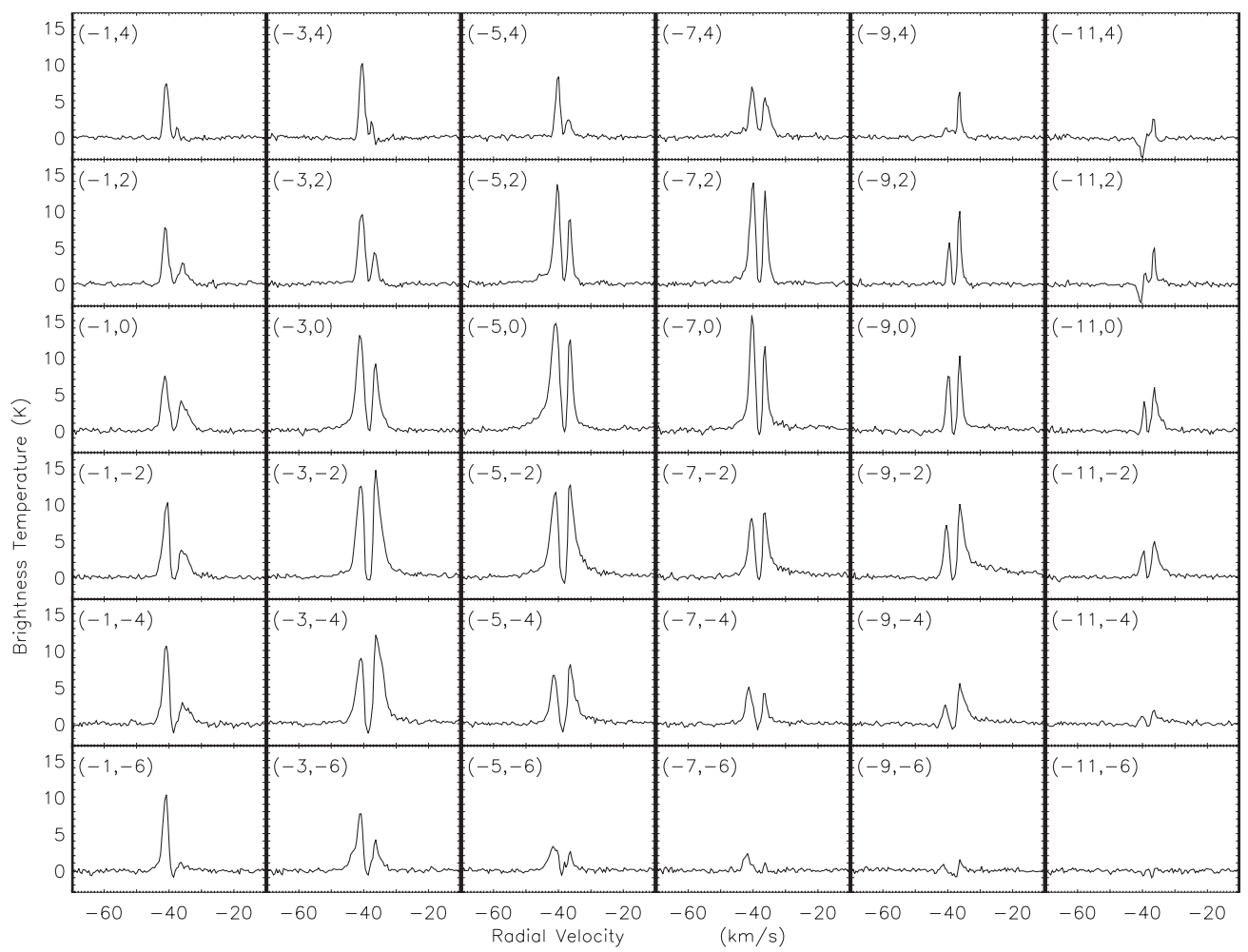

Figure 5. Grid of spectra for the $\mathrm{HCO}^{+}(3-2)$ line emission. The region covered is marked as the black rectangle in Figure 2(b). The pair of numbers in parentheses at top left in each of the spectra gives the offset in units of arcseconds with respect to the reference center.
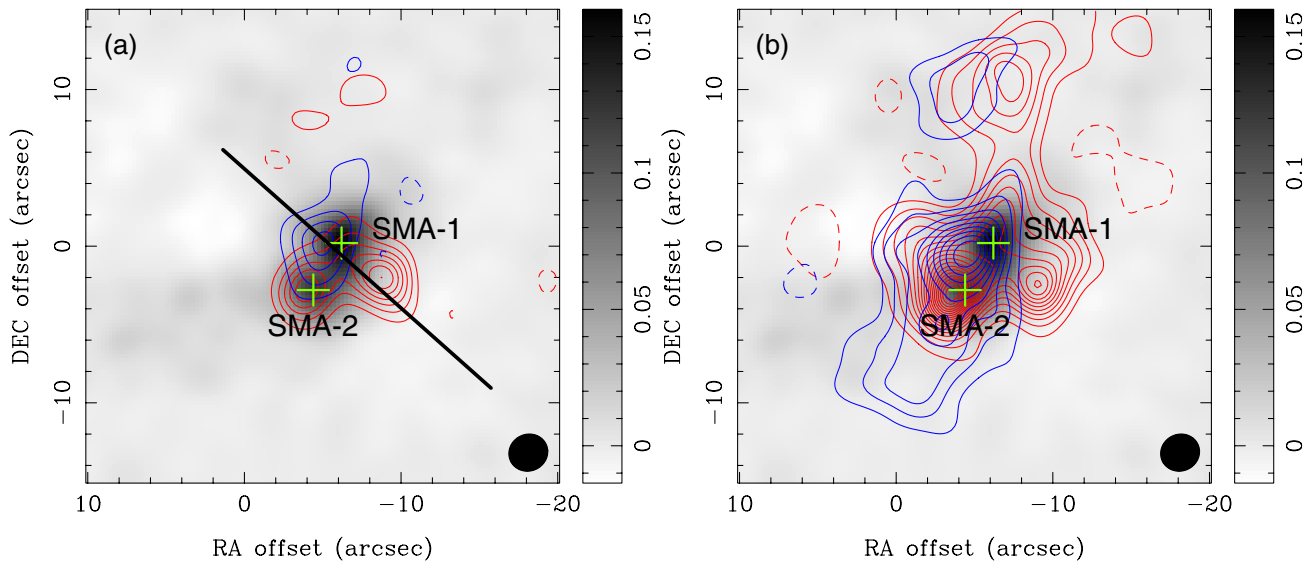

Figure 6. (a) The high-velocity outflow map in $\mathrm{HCO}^{+}(3-2)$, integrated from the blue wing in the velocity range $(-57.9,-45.3) \mathrm{km} \mathrm{s}^{-1}$ (blue contours) and the red wing in $(-31.8,-18.8) \mathrm{km} \mathrm{s}^{-1}$ (red contours). Contours are $(-1,1,2,3,4 \ldots) \times 4 \sigma ; 1 \sigma=0.2 \mathrm{Jy} \mathrm{beam}^{-1} \mathrm{~km} \mathrm{~s}^{-1}$. The thick straight line is the same as in Figure $4(\mathrm{a})$. (b) The low-velocity outflow map in $\mathrm{HCO}^{+}(3-2)$, integrated from the blue wing in $(-44.9,-41.7) \mathrm{km} \mathrm{s}^{-1}$ (blue contours) and the red wing in $(-36.3,-32.3) \mathrm{km} \mathrm{s}^{-1}$ (red contours). Contours are $(-1,1,2,3,4 \ldots) \times 5 \sigma ; 1 \sigma=0.16 \mathrm{Jy} \mathrm{beam}^{-1} \mathrm{~km} \mathrm{~s}^{-1}$. For both the figures the FWHM beam is $2^{\prime \prime} .5 \times 2^{\prime \prime} .3, \mathrm{P} . \mathrm{A} .=-65^{\circ}$. For both figures the background is the $1.1 \mathrm{~mm}$ continuum image, and the wedges are in units of $\mathrm{Jy}_{\text {beam }}{ }^{-1}$.

lobe and 3".4 for the red lobe) than the lobes in $\mathrm{HCN}(3-2)$. This could be explained if $\mathrm{HCN}(3-2)$ emission is excited close to bow shocks, while $\mathrm{HCO}^{+}(3-2)$ traces colder and more extended gas which is farther from bow shocks. Another possible reason is that the emission of the high-velocity outflow lobes might be blended by the $\mathrm{HCO}^{+}(3-2)$ emission from a large-scale outflow (Zhu et al. 2010) and other dynamical components associated with SMA-2. Indeed, in Figure 6(a) a redshifted $\mathrm{HCO}^{+}(3-2)$ component is found close to SMA-2 at high velocity. The same component is also marginally detected in $\mathrm{HCN}(3-2)$ (see Figure 4(a)), and it might be an outflow component associated with SMA-2.

The low-velocity line wings of the $\mathrm{HCO}^{+}(3-2)$ spectra were also integrated with the velocity ranges $(-44.9,-41.7) \mathrm{km} \mathrm{s}^{-1}$ and $(-36.3,-32.3) \mathrm{km} \mathrm{s}^{-1}$ for blue and red lobes, respectively. The velocity ranges have lower velocity limits similar to the ones used in a previous $\mathrm{HCO}^{+}(1-0)$ study (Zhu et al. 2010). As Figure 6(b) shows, the distribution of blueshifted and redshifted low-velocity $\mathrm{HCO}^{+}(3-2)$ gas is consistent with the results of $\mathrm{HCO}^{+}(1-0)$, implying a possible large-scale outflow in a NW-SE direction.

$$
\text { 3.3. } \mathrm{N}_{2} \mathrm{H}^{+}(3-2)
$$

A transition of protonated nitrogen, $\mathrm{N}_{2} \mathrm{H}^{+}(3-2)$ at $\nu_{0}=$ $279.512 \mathrm{GHz}$ was included in the LSB of the 2008 December observations. Figure 2(c) shows the integrated intensity map of $\mathrm{N}_{2} \mathrm{H}^{+}(3-2)$, indicating multiple $\mathrm{N}_{2} \mathrm{H}^{+}(3-2)$ emission clumps which form a curved filamentary emission region. Among 


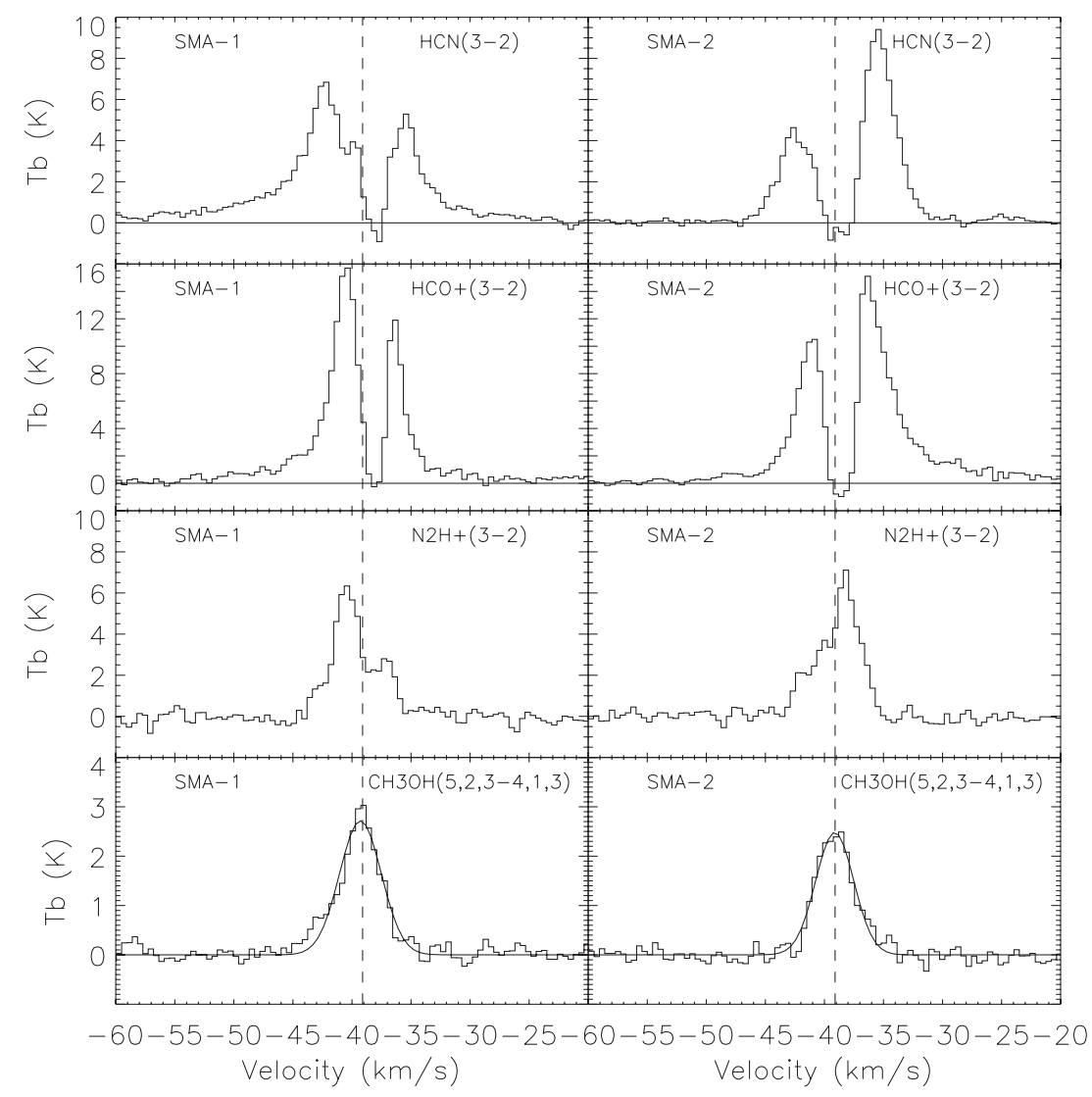

Figure 7. Spectra of $\mathrm{HCN}(3-2), \mathrm{HCO}^{+}(3-2), \mathrm{N}_{2} \mathrm{H}^{+}(3-2)$, and $\mathrm{CH}_{3} \mathrm{OH}\left(5_{2,3}-4_{1,3}\right)$ (from top to bottom) at SMA-1 (left panels) and SMA-2 (right panels). The vertical dashed line in the two figures denotes the peak velocities of the $\mathrm{CH}_{3} \mathrm{OH}\left(5_{2,3}-4_{1,3}\right)$ lines $\left(-39.1 \mathrm{~km} \mathrm{~s}^{-1}\right)$, which is used as the systemic velocity throughout this paper.

the numerous $\mathrm{N}_{2} \mathrm{H}^{+}(3-2)$ emission peaks, the two strongest emission peaks appear to be located near SMA-1 and SMA2 with a displacement of $\sim 0^{\prime} .5$ from the continuum peaks, suggesting that a close relationship exists between the dense molecular gas and the protostellar cores.

The bright $\mathrm{N}_{2} \mathrm{H}^{+}(3-2)$ emission region is located $\mathrm{SW}$ of the double continuum cores, opposite to the locations of the bright emission ridge of $\mathrm{HCO}^{+}(3-2)$. Emission at the locations of SMA-1 and SMA-2 might be influenced by excitation or chemical conditions. The primary formation routes for both $\mathrm{HCO}^{+}$and $\mathrm{N}_{2} \mathrm{H}^{+}$involve reactions with $\mathrm{H}_{3}^{+}$. For a higher $\mathrm{CO}$ abundance, the main formation route is the reaction between $\mathrm{CO}$ and $\mathrm{H}_{3}^{+}$in forming $\mathrm{HCO}^{+}$. When the $\mathrm{CO}$ abundance gets low enough, the reaction between $\mathrm{H}_{3}^{+}$and $\mathrm{N}_{2}$ in forming $\mathrm{N}_{2} \mathrm{H}^{+}$becomes the main mechanism for the removal of $\mathrm{H}_{3}^{+}$ (Jorgensen et al. 2004b). In addition, as CO returns to the gas phase from a freezing-out phase, destruction of $\mathrm{N}_{2} \mathrm{H}^{+}$through reactions with $\mathrm{CO}$ becomes the dominant removal mechanism for $\mathrm{N}_{2} \mathrm{H}^{+}$. The possible anti-correlation of the bright emission regions between $\mathrm{N}_{2} \mathrm{H}^{+}(3-2)$ and $\mathrm{HCO}^{+}(3-2)$ near the protostellar cores might reflect a gradient of the $\mathrm{CO}$ abundance across the W3-SE core.

The line profiles of $\mathrm{N}_{2} \mathrm{H}^{+}(3-2)$ spectra toward SMA-1 and SMA-2 are shown in Figure 7. We notice the skewness of the line profiles, i.e., at the position of SMA-1, the $\mathrm{N}_{2} \mathrm{H}^{+}(3-2)$ spectrum mimics a "blue profile," while toward SMA-2, $\mathrm{N}_{2} \mathrm{H}^{+}(3-2)$ is like a "red profile." The main hyperfine structures of the transition $J=3-2$ for $\mathrm{N}_{2} \mathrm{H}^{+}$spread over a frequency range of only a few $100 \mathrm{kHz}\left(100 \mathrm{kHz} \sim 0.1 \mathrm{~km} \mathrm{~s}^{-1}\right)$, while the velocity difference between the two emission peaks of the $\mathrm{N}_{2} \mathrm{H}^{+}(3-2)$ spectrum toward SMA-1 is $\sim 5.0 \mathrm{~km} \mathrm{~s}^{-1}$. Therefore, it is unlikely that the observed skewness in the $\mathrm{N}_{2} \mathrm{H}^{+}(3-2)$ line profile is due to hyperfine structure.

In order to model the overall kinematics of the molecular filament in the region, a centroid velocity map of $\mathrm{N}_{2} \mathrm{H}^{+}(3-2)$ was made (see Figure 8(a)) by calculating $v_{\text {cen }}=\int v I_{v} d v / \int I_{v} d v$ at each position. Here $v_{\text {cen }}$ is the centroid velocity (intensityweighted mean velocity) and $I_{v}$ is the intensity of the spectrum at a given velocity $v$. In general, the distribution of the centroid velocity shows a gradient of $30 \pm 10 \mathrm{~km} \mathrm{~s}^{-1} \mathrm{pc}^{-1}$ over a spatial scale of $\sim 20^{\prime \prime}$, i.e., the gas in the $\mathrm{NW}$ is redshifted while that in the SE is blueshifted. This velocity structure appears to be similar to the large-scale velocity structure traced by $\mathrm{HCO}^{+}(1-0)$ that is believed to be a result of outflow (Zhu et al. 2010).

$$
\text { 3.4. } \mathrm{CH}_{3} \mathrm{OH}\left(5_{2,3}-4_{1,3}\right)
$$

The methanol transition $\mathrm{CH}_{3} \mathrm{OH}\left(5_{2,3}-4_{1,3}\right)$ at $v_{0}=$ $266.838 \mathrm{GHz}$ was observed in the LSB in the 2008 October observations. Figure 2(d) shows the integrated intensity map of this transition. The two main $\mathrm{CH}_{3} \mathrm{OH}\left(5_{2,3}-4_{1,3}\right)$ emission peaks coincide with the two protostellar cores SMA-1 and SMA-2 within the uncertainty in position $\left(\sim 0^{\prime} .2\right)$, indicating that this line traces the densest part of the cores and could be used to determine the systemic velocity. The methanol emission appears to arise mainly from the two protostellar cores as well as the high-velocity outflow from SMA-1. In addition, in region B, a weak signal of $\mathrm{CH}_{3} \mathrm{OH}\left(5_{2,3}-4_{1,3}\right)$ is detected at a $3 \sigma$ level. No significant emission from $\mathrm{CH}_{3} \mathrm{OH}\left(5_{2,3}-4_{1,3}\right)$ was detected toward region $\mathrm{C}$. The distribution of the centroid velocity in 

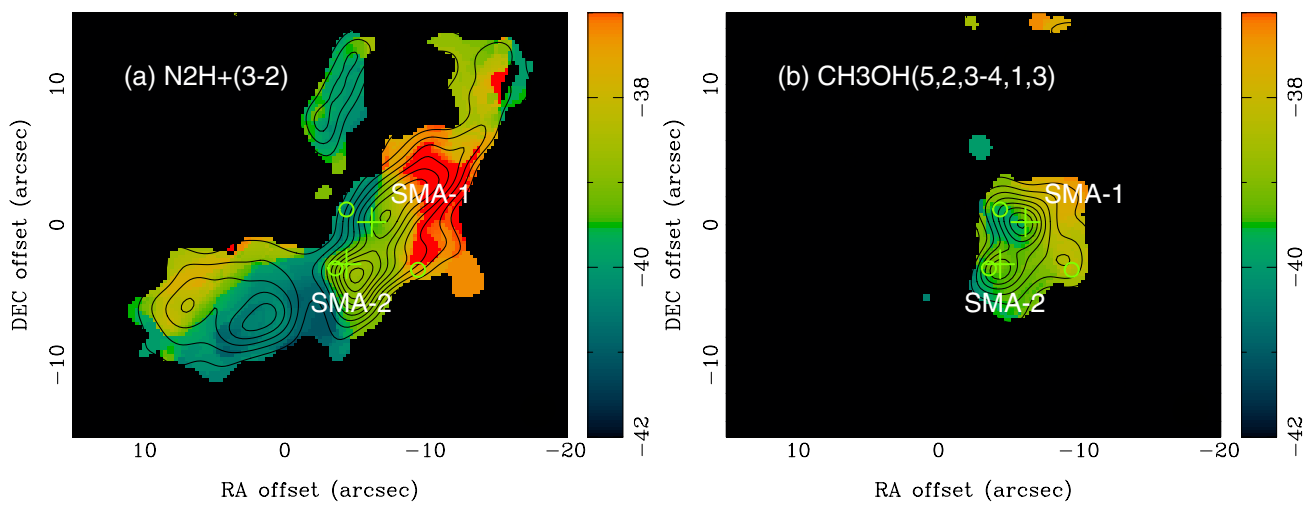

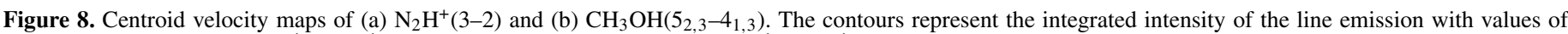

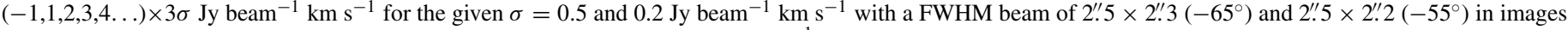
(a) and (b), respectively. The color wedge scales the radial velocity in units of $\mathrm{km} \mathrm{s}^{-1}$.

$\mathrm{CH}_{3} \mathrm{OH}$ emission (Figure 8(b)) has no significant velocity gradients, although at the northwest boundary and the position of A-SW the centroid velocity is slightly redshifted.

\subsection{Comparison of Molecular Line Spectra Toward SMA-1 and SMA-2}

Figure 7 shows the spectra from the peak positions of SMA-1 and SMA-2 in the lines of $\mathrm{HCN}(3-2), \mathrm{HCO}^{+}(3-2), \mathrm{N}_{2} \mathrm{H}^{+}(3-2)$, and $\mathrm{CH}_{3} \mathrm{OH}\left(5_{2,3}-4_{1,3}\right)$, respectively. In general, double-peaked profiles appear to characterize the optically thick lines toward both cores from different molecules. For a given molecular line, the double-peaked profile of SMA-1 is dominated by the blueshifted feature, whereas in SMA-2, the redshifted peak is stronger than the blueshifted one. For a given core, the separation between the peaks of the double-peaked line profile appears to vary. For example, in the case of SMA-1, the velocity separations between the two spectral peaks vary significantly from $7.6 \mathrm{~km} \mathrm{~s}^{-1}$ for the $\mathrm{HCN}(3-2)$ line to $4.0 \mathrm{~km} \mathrm{~s}^{-1}$ for both the $\mathrm{HCO}^{+}(3-2)$ and $\mathrm{N}_{2} \mathrm{H}^{+}(3-2)$ lines, suggesting that different molecular lines may trace different dynamic processes or chemistry in a protostellar core. For the $\mathrm{HCN}(3-2)$ line from SMA-1, the large velocity separation of $7.6 \mathrm{~km} \mathrm{~s}^{-1}$ between the double emission peaks is probably due to the high-velocity bipolar outflow because the $\mathrm{HCN}(3-2)$ line is the best tracer of the shocked and compressed medium among the four observed molecular lines shown in this paper.

In contrast to the double spectral peaks observed in $\mathrm{HCN}(3-2)$ and $\mathrm{HCO}^{+}(3-2)$, the hot molecular line $\mathrm{CH}_{3} \mathrm{OH}\left(5_{2,3}-4_{1,3}\right)$ shows a single-peaked profile toward both the protostellar cores. Gaussian fits to the spectra show that $\mathrm{CH}_{3} \mathrm{OH}\left(5_{2,3}-4_{1,3}\right)$ peaks at $-39.1 \pm 0.1 \mathrm{~km} \mathrm{~s}^{-1}$ for both SMA-1 and SMA-2. We use this as the systemic velocity in this paper, i.e., $V_{\text {sys }}=-39.1 \mathrm{~km} \mathrm{~s}^{-1}$. The apparent peak velocities of the spectra have a difference of $\sim 0.4 \mathrm{~km} \mathrm{~s}^{-1}$, equivalent to the width of one channel. Figure 8 (b) also suggests a difference $\sim 0.5 \mathrm{~km} \mathrm{~s}^{-1}$ between the centroid velocities of $\mathrm{CH}_{3} \mathrm{OH}\left(5_{2,3}-4_{1,3}\right)$ toward the two cores. However, this inconsistency could be due to the slightly asymmetric $\mathrm{CH}_{3} \mathrm{OH}\left(5_{2,3}-4_{1,3}\right)$ line profile toward SMA-2 and contamination from the complex kinematics in the W3-SE region (e.g., the outflow from SMA-1). Thus, we conclude that there is no significant difference between the radial velocities of $\mathrm{CH}_{3} \mathrm{OH}\left(5_{2,3}-4_{1,3}\right)$ from SMA-1 and SMA-2.

An emission bump at $-40.2 \mathrm{~km} \mathrm{~s}^{-1}$ is present in the spectral dip between the double HCN(3-2) emission peaks toward SMA1 (see left top panel in Figure 7). The velocity of the emission bump appears to correspond to the velocity of the blueshifted peak in both the $\mathrm{HCO}^{+}(3-2)$ and $\mathrm{N}_{2} \mathrm{H}^{+}(3-2)$ lines, which is blueshifted with respect to the systemic velocity. In addition, the redshifted dip between the two main emission peaks appears to be an absorption feature and the intensity becomes negative $(\sim 5 \sigma)$ at $-37.6 \mathrm{~km} \mathrm{~s}^{-1}$. We will discuss these spectral features in Section 4.2.1.

\section{DISCUSSION}

\subsection{High-velocity Bow-shock Outflow in HCN(3-2)}

Two possible mechanisms for accelerating molecular gas in outflows from protostellar cores are stellar winds and highly collimated jets (e.g., Downes \& Ray 1999). The broad velocity distribution with a power law in both blueshifted and redshifted $\mathrm{HCN}(3-2)$ line wings and the morphology of the high-velocity $\mathrm{HCN}(3-2)$ lobes suggests that high-velocity $\mathrm{HCN}(3-2)$ emission is excited at jet-driven bow shocks. An outflow model with jet-driven bow shocks has been studied both analytically and numerically (e.g., Downes \& Ray 1999; Lee et al. 2000). In the case of W3-SE, a position-velocity (P-V) diagram along the major axis of the bipolar outflow observed in the $\mathrm{HCN}(3-2)$ line (Figure 9) shows similar characteristics to that predicted from the bow-shock model. The broad blueshifted and redshifted velocity gas components show significant offsets of $2^{\prime \prime} .2 \pm 0.2$ and $5^{\prime \prime} .0 \pm 0.2$ from SMA-1, providing evidence that the highvelocity $\mathrm{HCN}(3-2)$ gas is indeed excited and accelerated at the bow-shocked sites outside of the protostellar core SMA-1.

To characterize the observed high-velocity outflow, we adopted a simple model of bow-shock outflow (Downes \& Ray 1999), in which the geometry and velocity of the molecular gas along the bow-shock surface can be worked out in analytical forms. Using the model illustrated in Figure 10, a cylindrical symmetric outflow radius $(r)$ and major axis $(z)$ plane are used following Downes \& Ray (1999). We derived equations for the four boundaries that outline the observed P-V diagram (see the Appendix). A power law $\left(z \sim|r|^{s}\right)$ with an index $s=2$ for the locus of the bow-shock surface is assumed in fitting the model to the observed P-V diagram of the bipolar outflow from SMA-1 as discussed below. The systemic velocity, $V_{\text {sys }}=-39.1 \mathrm{~km} \mathrm{~s}^{-1}$, and the projected angular offsets of the blueshifted and redshifted bow-shock tips $\left(a_{B} \approx 2^{\prime \prime} .2\right.$ and $\left.a_{R} \approx 5^{\prime \prime} .0\right)$ from the outflow origin have been determined directly from the peak velocity of the hot molecular line $\mathrm{CH}_{3} \mathrm{OH}\left(5_{2,3}-4_{1,3}\right)$ (Figure 7) and the $\mathrm{HCN}(3-2)$ outflow map (Figure 4(a)), respectively. The remaining two free parameters $\alpha$ (inclination angle) and $v$ (the 


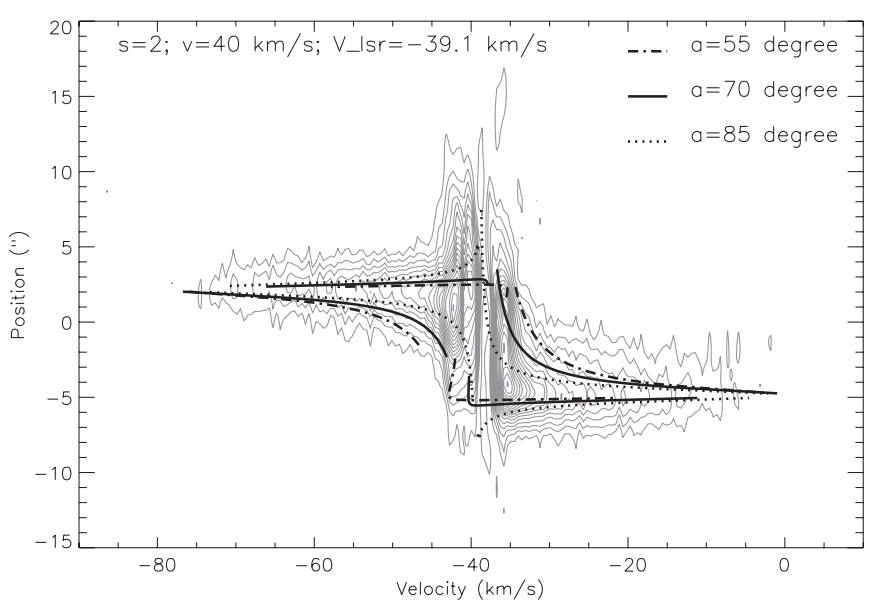

Figure 9. Position-velocity (P-V) diagram of $\mathrm{HCN}(3-2)$ along the outflow axis as indicated with the straight line in Figure 4. The origin of the position axis corresponds to the position of SMA-1 projected onto the axis. Contours are 0.2 , $0.4,0.6,0.8, \ldots \mathrm{Jy} \mathrm{beam}^{-1}$. The angular and velocity resolutions are 2.7 and $0.45 \mathrm{~km} \mathrm{~s}^{-1}$, respectively. The observed P-V diagram is fitted with the bowshocked outflow model as discussed in the Appendix assuming the systemic velocity in the LSR frame $\left(V_{\text {sys }}=-39.1 \mathrm{~km} \mathrm{~s}^{-1}\right)$, the velocity of the bow shock $\left(v=40 \mathrm{~km} \mathrm{~s}^{-1}\right)$, and the spectral index of the power law for the bowshock surface as a function of radius $(s=2)$. The dot-dashed, solid, and dashed lines stand for the model curves corresponding to the three different values of inclination angle $\left(\alpha=55^{\circ}, 70^{\circ}\right.$, and $\left.85^{\circ}\right)$. The curve with $\alpha=70^{\circ}$ appears to represent the best fit. Note that the bow-shock outflow model shows a good fit to the high-velocity gas. The low-velocity gas is probably disturbed by additional processes other than outflow. For example, the emission in the top left quadrant in the velocities of $(-40,-45) \mathrm{km} \mathrm{s}^{-1}$ and the position offsets of $\left(5^{\prime \prime}, 6^{\prime \prime}\right)$ are likely from the infalling gas or the gas in the molecular envelope, which is not related to the bow-shock outflow.

velocity of the bow shock with respect to the origin of the outflow) were assumed to have equal values for the blueshifted and redshifted lobes, and they can be determined by comparing the observed P-V diagram and the curve from the bow-shocked outflow model. Figure 9 shows the observed P-V diagram as contours and the curves derived from the bow-shock outflow model as thick lines. A shock velocity $v=40 \mathrm{~km} \mathrm{~s}^{-1}$ and three different inclination angles $\alpha=55^{\circ}, 70^{\circ}$, and $85^{\circ}$ were used to generate the curves. It appears that the model with $v=40 \pm$ $10 \mathrm{~km} \mathrm{~s}^{-1}$ and $\alpha=70^{\circ} \pm 5^{\circ}$ best fits the overall line wings of the $\mathrm{P}-\mathrm{V}$ diagram. To estimate the uncertainty $(1 \sigma)$ in the values of $v$ and $\alpha$, we added a small offset to cause the derived model curve to offset by one contour interval of the P-V diagram $(3 \sigma)$. The inferred shock velocity of the bipolar outflow implies a dynamic age of $3 \times 10^{3} \mathrm{yr}$ for the protostellar core SMA-1, which is an order of magnitude younger than that inferred for the possible large-scale molecular outflow in the region (Zhu et al. 2010), indicating that the source(s) embedded in SMA-1 is probably very young. On the other hand, SMA-1 is known to be associated with a mid-IR source (Zhu et al. 2010) which implies the existence of a heating source in SMA-1. We offer two possible explanations, i.e., the protostar in SMA-1 has a large mass and evolves more rapidly than classic low-mass stars, or there are multiple young stellar objects (YSOs) embedded in SMA-1 and the compact outflow and the mid-IR source are actually associated with different YSOs.

\subsection{Infalling Gas Toward SMA-1 \\ 4.2.1. Possible Infall Signature}

The blue-dominated double-peaked profile of the $\mathrm{HCO}^{+}(1-0)$ spectrum toward core A observed using CARMA at 6" resolu-

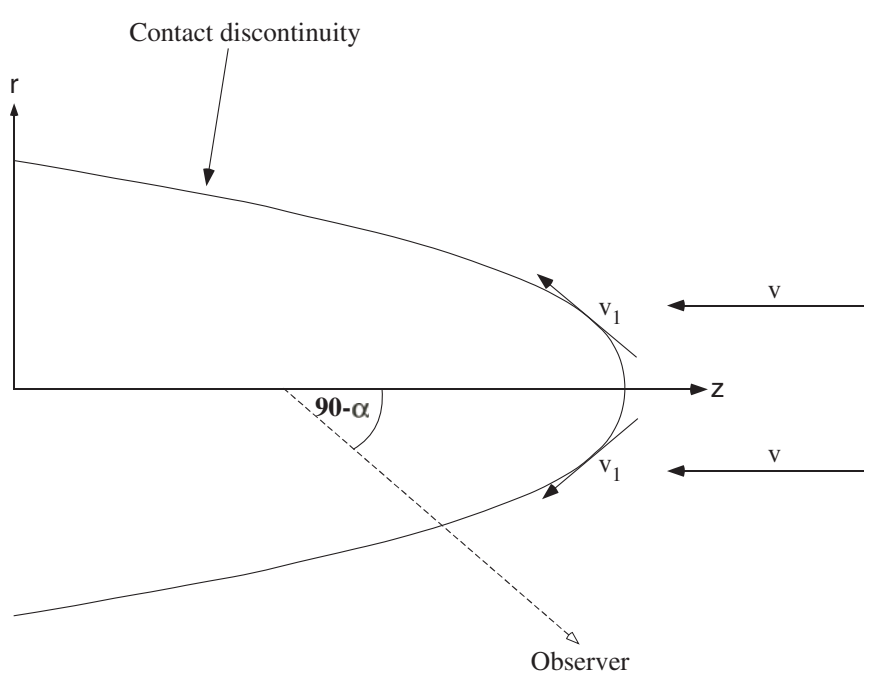

Figure 10. Sketch illustrating the geometry of the bow-shock outflow model, which is taken from Figure 6 in Downes \& Ray (1999).

tion was fitted using a model with both outflow and infall components (Zhu et al. 2010). The fit suggests that outflow contributes significantly to the spectral profile, particularly in the highvelocity wings. The high-velocity outflow lobes from SMA-1 are separated from the protostellar core in the SMA HCN(3-2) observations at $(2$.'5 $\times 2$ '. 3$)$ resolution. Contamination from the high-velocity outflow in the spectra toward the cores has been reduced to a level where an inverse P-Cygni profile, a signature of infall, would become noticeable if it exists.

The HCN(3-2) spectra (Figure 3), show blueshifted emission at $-40.2 \mathrm{~km} \mathrm{~s}^{-1}$ toward SMA-1 and the adjacent region, with a sharp dip in the emission at $-37.6 \mathrm{~km} \mathrm{~s}^{-1}$. The systemic velocity of $-39.1 \mathrm{~km} \mathrm{~s}^{-1}$ lies between the two spectral features. The complexity of the $\mathrm{HCN}(3-2)$ line profile toward SMA-1 might be due to a superimposition of an inverse P-Cygni profile and a broader double-peaked profile from the high-velocity bipolar outflow. Such an explanation is consistent with the hypothesis that gas is infalling to SMA-1 (Zhu et al. 2010).

Figure 7 shows that toward SMA-1 the intensity of the $\mathrm{HCN}(3-2)$ dip at $-37.6 \mathrm{~km} \mathrm{~s}^{-1}$ becomes negative. The spectral dip could be partially caused by unsampled short spacings. However, in our observations the shortest projected baseline is $5.4 \mathrm{k} \lambda$, corresponding to a spatial scale of $\sim 38^{\prime \prime}$. Thus, for the spectrum toward SMA-1 with a beam size $(2$. $5 \times 2$ 2.3), missing flux should not be a severe problem. We can roughly estimate the short-spacing effect using the previous study of CARMA data. For the $\mathrm{HCO}^{+}(1-0)$ spectra at the peak position, the flux density of the spectral dip was raised to $0.5 \mathrm{Jy}^{\text {beam }}{ }^{-1}$ from 0.0 Jy beam $^{-1}$ after combining with single-dish data. Assuming that the missing flux density of $0.5 \mathrm{Jy} \mathrm{beam}^{-1}$ is evenly distributed in a structure on a scale of the CARMA beam $(6.3 \times 5.7)$, and toward SMA-1 the flux density of $\operatorname{HCN}(3-2)$ is the same as $\mathrm{HCO}^{+}(1-0)$, the missing flux in $\mathrm{HCN}(3-2)$ at the velocity of the dip is less than 0.1 Jy within the SMA beam. On the other hand, the intensity of $\mathrm{HCN}(3-2)$ toward SMA-1 is $\sim-1 \mathrm{~K}(\sim-0.4$ $\mathrm{Jy} \mathrm{beam}^{-1}$ ) at the spectral dip. Therefore, in the case of W3-SE the missing flux caused by unsampled short spacings has only a small influence in the core region on a scale of the beam size, and the observed negative intensity for the $\mathrm{HCN}(3-2)$ spectral dip is likely to be real.

If the emission bump is explained as an inverse P-Cygni feature, the infalling gas associated with SMA-1 appears to be 
confined to a region $\sim 6^{\prime \prime}(\sim 12,000 \mathrm{AU})$ with velocities in a range of $\left(-40\right.$ to $\left.-38 \mathrm{~km} \mathrm{~s}^{-1}\right)$ that is close to the systemic velocity of the protostellar core. We made an integrated intensity map of this emission in a velocity range from -40.0 to $-39.5 \mathrm{~km} \mathrm{~s}^{-1}$ (Figure 4(b)). This image clearly shows that the $\operatorname{HCN}(3-2)$ feature is associated with SMA-1; thus SMA-1 is likely to be the gravitational center. Weak HCN(3-2) emission to the SE of SMA-1 at a similar radial velocity could blend with the observed $\mathrm{HCN}(3-2)$ spectra from SMA-1.

\subsubsection{A General Model Including Both Infall and Outflow}

In order to separate the possible inverse P-Cygni (infall) feature from the outflow, we adopted a characteristic model with a continuum core in the middle comprised of two layers of infalling gas and two layers of outflowing gas located outside of the infall core, which is hereafter referred to as a four-layer model (Myers et al. 1996; Di Francesco et al. 2001; Attard et al. 2009). The schematic configuration of the model is illustrated in Figure 4 of Attard et al. (2009).

In the following discussion, the subscripts $f, r, B, R$, and $c$ are used to represent the front infall layer, rear infall layer, blueshifted outflow layer, redshifted outflow layer, and the continuum core, respectively. On the assumption of homogeneous and isothermal layers of gas, the line intensity or brightness temperature from the "four-layer complex" can be solved from the radiative transfer equation for given excitation temperatures $T_{f}$ and $T_{r}$ of the front and rear layers of infall, $T_{\text {out }}$ of both the outflow layers, and $T_{c}$ of the continuum emission. The line brightness temperature, after subtracting the continuum in which the contribution from the cosmic background radiation is negligible, can be expressed as

$$
\begin{aligned}
\Delta T_{L}= & J\left(T_{f}\right)\left[\left(1-\frac{J\left(T_{c}\right) f_{c}\left(1-e^{-\tau_{c}}\right)}{J\left(T_{f}\right)}\right)\left(1-e^{-\tau_{f}}\right)\right. \\
& \left.+\frac{J\left(T_{r}\right)}{J\left(T_{f}\right)}\left(1-e^{-\tau_{r}}\right) e^{-\tau_{f}-\tau_{c}}\right] e^{-\tau_{B}} \\
& +J\left(T_{\text {out }}\right)\left[\left(1-\frac{J\left(T_{c}\right) f_{c}\left(1-e^{-\tau_{c}}\right)}{J\left(T_{\text {out }}\right)}\right)\left(1-e^{-\tau_{B}}\right)\right. \\
& \left.+\left(1-e^{-\tau_{R}}\right) e^{-\tau_{B}-\tau_{f}-\tau_{c}-\tau_{r}}\right]
\end{aligned}
$$

where $J(T)=\frac{h v}{k}\left(e^{h v / k T}-1\right)^{-1}$ is the radiation temperature of each layer and $f_{\mathrm{c}}$ is the beam filling factor for the continuum emission. Equation (1) shows that, in the four-layer model, the resultant line profile can be expressed as two separate terms. The term in the first bracket on the right side of Equation (1) describes the line profile of the infalling gas, corresponding to an inverse P-Cygni profile in the case of $J\left(T_{c}\right) f_{c} \tau_{c}>J\left(T_{f}\right)$. The infall line profile is attenuated by the absorption in the blueshifted outflow $\left(e^{-\tau_{B}}\right)$. The term in the second bracket corresponds to the line profile from the bipolar outflow showing a classical PCygni profile if $J\left(T_{c}\right) f_{c} \tau_{c}>J\left(T_{\text {out }}\right)$ or a red-dominated doublepeaked profile if $J\left(T_{c}\right) f_{c} \tau_{c}<J\left(T_{\text {out }}\right) f_{\text {out }}$. The emission from the redshifted outflow layer is attenuated by the absorbing gas in both the infall layers and the blueshifted outflow as well as the continuum dust $\left(e^{-\tau_{f}-\tau_{r}-\tau_{B}-\tau_{c}}\right)$.

Each of the optical depths in the four layers can be modeled using a Gaussian function with a mean velocity and a velocity dispersion for infall $\left(V_{\text {in }}, \sigma_{\text {in }}\right)$ and outflow $\left(V_{\text {out }}, \sigma_{\text {out }}\right)$ assuming the systemic velocity of $V_{\text {sys }}$. Therefore, a total of 14 parameters, namely the five kinematic parameters $\left(V_{\text {in }}, V_{\text {out }}, \sigma_{\text {in }}, \sigma_{\text {out }}\right.$, and $\left.V_{\text {sys }}\right)$ along with four excitation temperatures $\left(T_{f}, T_{r}, T_{\text {out }}\right.$, and $\left.T_{c}\right)$ and five optical depths $\left(\tau_{f}, \tau_{r}, \tau_{B}, \tau_{R}\right.$, and $\left.f_{c} \tau_{c}\right)$ are required to characterize the physical conditions of the gas in each layer.

\subsubsection{Fitting the HCN(3-2) Spectra}

We made a tentative fit to the observed $\mathrm{HCN}(3-2)$ spectra with the model described by Equation (1). In the case of W3$\mathrm{SE}$, the quantities for the continuum emission $\left(T_{c}=40 \mathrm{~K}\right.$ and $\left.\tau_{c}=0.5\right)$ can be determined from the SED as discussed by Zhu et al. (2010) assuming that the ratio of 2:1 in dust content between SMA-1 and SMA-2 is proportional to the ratio of the total between the two sources. Therefore, 13 free parameters are needed.

In order to fit the four-layer model to the observed $\mathrm{HCN}(3-2)$ line profiles, we first assumed the two outflow layers are optically thin at low velocities and fit the low-velocity part (from -43.5 to $-34.1 \mathrm{~km} \mathrm{~s}^{-1}$ ), or the assumed infall part, with the least-square approach. The high-velocity parts of the blue $\left(<-43.5 \mathrm{~km} \mathrm{~s}^{-1}\right)$ and red $\left(>-34.1 \mathrm{~km} \mathrm{~s}^{-1}\right)$ wings of $\mathrm{HCN}(3-2)$ are related to the bow shock, and could not be fitted with Equation (1). Therefore, they are fitted separately with a powerlaw profile $\left(\propto\left|V-V_{\text {sys }}\right|^{-\gamma}\right)$. The maximum intensities of the power-law line wings are set to be at -43.5 and $-34.1 \mathrm{~km} \mathrm{~s}^{-1}$ for blue and red wings, respectively, and the values are obtained from fitting the low-velocity line profile with Equation (1).

The best-fit results are shown in Figure 11(a), which gives an infall velocity $0.9 \mathrm{~km} \mathrm{~s}^{-1}$. The derived excitation temperatures are $6_{-2}^{+1}$ and $11 \pm 3 \mathrm{~K}$ for the front and rear layers, respectively. The best fits for the 13 free parameters in the final stage were adjusted by eye to achieve a minimum residual. The errors $(1 \sigma)$ were assessed by adding an offset $(3 \sigma)$ to a best-fitted quantity with other parameters fixed, to increase the offset of the best-fitted curve from the observed profile (or a factor of three increase in the residual). The higher excitation temperature for the rear layer might suggest the presence of a temperature gradient in radius (temperature increases as it gets close to the protostar). Thus, an observer receives emission from a colder part in the front and a warmer part in the rear layers, considering the optical depth effect. On the other hand, the excitation temperature of the infalling gas is also constrained by the observed brightness temperature in the presence of an inverse P-Cygni profile. As both angular and spectral resolution improve, observations with interferometer arrays at millimeter and submillimeter wavelengths will place more meaningful constraints on the infalling gas in protostellar cores. For the bipolar outflow in SMA-1, the gas components in both the blueshifted and redshifted outflows are optically thin $\left(\tau_{\text {out }} \sim\right.$ 0.1 ) with excitation temperatures of $\sim 75 \mathrm{~K}$, which are much higher than the beam-averaged peak brightness temperature $(\sim 8 \mathrm{~K})$ of $\mathrm{HCN}(3-2)$. As for the high-velocity parts of the spectrum, the power-law index $\gamma$ for the blue and red lobes of the outflow is 2.0 and 2.5 , respectively. The difference in the two values could be due to a slight difference in the directions of the ejected blueshifted and redshifted high-velocity gas components.

Fitting the four-layer model to the spectral profile of SMA2 suggests that a bipolar outflow might also be present in this protostellar core (see Figure 11(b)). In comparison, the expected outflow from SMA-2 has a similar optical depth and excitation temperature to those derived from SMA-1 but it appears at an earlier stage. The observed line profile in the low-velocity range 

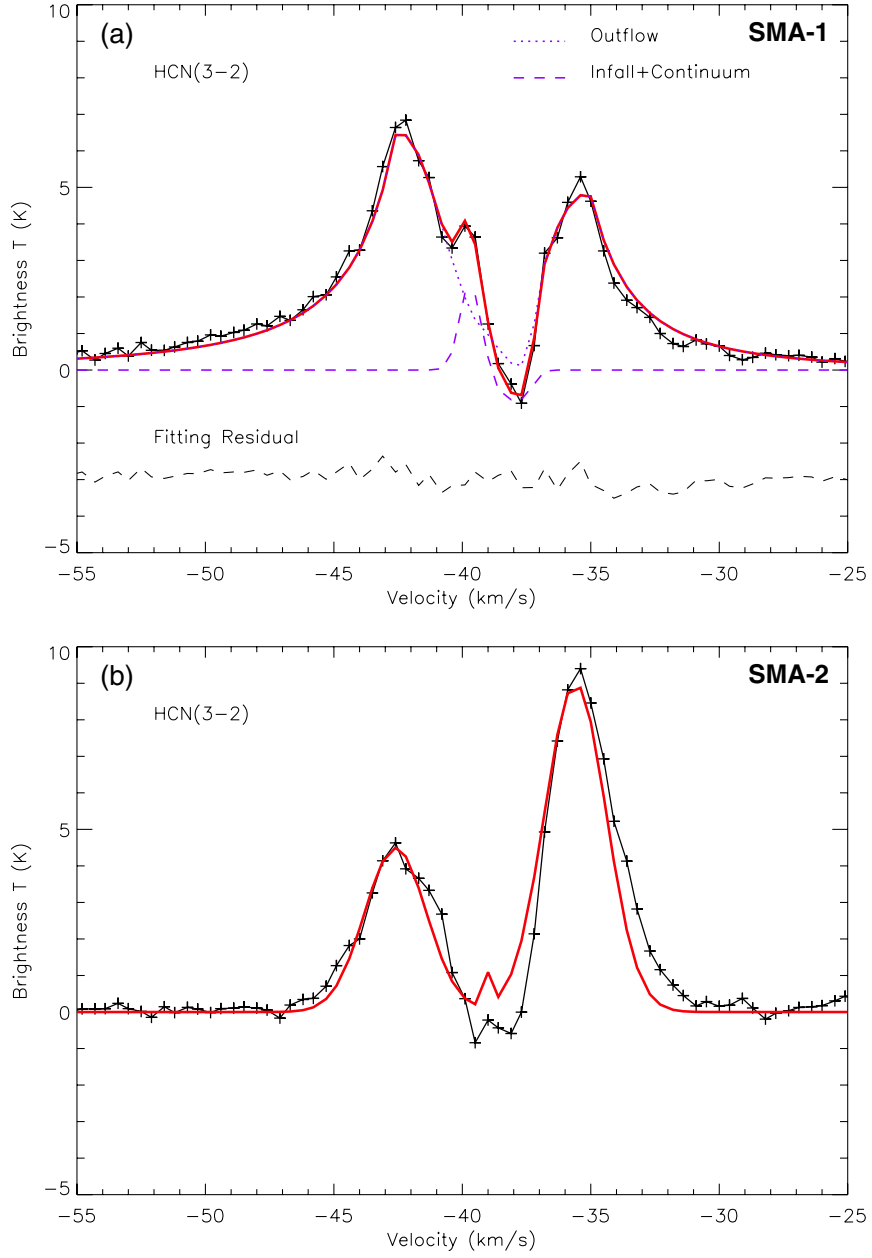

Figure 11. (a) The observed HCN(3-2) spectral profile toward SMA-1 (solid black curve) is fitted with the four-layer model (red curve) as described by Equation (1), which is discussed in Section 4.2.3. The best fit gives the optical depths and excitation temperature for the infall gas in the front and rear layers $\left(\tau_{\mathrm{f}}=5.0, \tau_{\mathrm{r}}=0.5, J\left(T_{\mathrm{f}}\right)=2 \mathrm{~K}, J\left(T_{\mathrm{r}}\right)=6 \mathrm{~K}\right)$, and the outflow gas $\left(\tau_{\mathrm{B}}=0.08, \tau_{\mathrm{R}}=0.06, J\left(T_{\text {out }}\right)=76 \mathrm{~K}\right)$, as well as the dust $\left(\tau_{\mathrm{c}}=0.5\right.$, $\left.J\left(T_{\mathrm{c}}\right) f_{\mathrm{c}}=7 \mathrm{~K}\right)$, respectively. The best-fitted velocities and velocity dispersions are $\left(V_{\text {in }}=0.9 \mathrm{~km} \mathrm{~s}^{-1}, \sigma_{\text {in }}=0.4 \mathrm{~km} \mathrm{~s}^{-1}\right)$ and $\left(V_{\text {out }}=3.6 \mathrm{~km} \mathrm{~s}^{-1}\right.$, $\sigma_{\text {out }}=1.6 \mathrm{~km} \mathrm{~s}^{-1}$ ) for the infall and the outflow, respectively. The blue dashed and dotted lines represent the best-fitted spectral components for the infall and the outflow described in Equation (2), respectively. The line wings are not fitted with the four-layer model; instead, they are simply fitted using a power-law profile with an index of 2 . The black dashed line is the residual of subtracting the best-fit model from the data. (b) The observed $\mathrm{HCN}(3-2)$ spectral profile toward SMA-2 is fitted to a model with outflow alone $\left(V_{\text {in }}=0 \mathrm{~km} \mathrm{~s}^{-1}\right)$ as shown by the red curve.

(A color version of this figure is available in the online journal.)

appears to be dominated by outflow emission and no obvious infall signatures are present toward SMA-2. SMA-2 is different from SMA-1 since it has no obviously associated mid-IR point sources. The relatively extended mid-IR emission adjacent to SMA-2 might be due to the external heating from the star formed in the $\mathrm{W} 3-\mathrm{SE}$ region.

We compared these fits with $\mathrm{HCN}(3-2)$ data with previous fits to CARMA $\mathrm{HCO}^{+}(1-0)$ data of lower angular resolution $\left(\sim 6^{\prime \prime}\right)$ using a similar four-layer model (Zhu et al. 2010). All the parameters from the $\mathrm{HCN}(3-2)$ fits (see the caption to Figure 11) are consistent with the previous $\mathrm{HCO}^{+}(1-0)$ fits, especially the infall velocity $\left(0.9 \mathrm{~km} \mathrm{~s}^{-1}\right)$. Unlike the $\mathrm{HCO}^{+}(1-0)$ fits, the $\mathrm{HCN}(3-2)$ fit suggests an inverse P-Cygni profile instead of a blue profile, which could be due to the larger contribution from the high-velocity outflow in $\mathrm{HCN}(3-2)$ and the significant improvement in angular resolution.

We found it is implausible to obtain consistent fits with Equation (1) for the entire W3-SE region, and the fitted values of $\tau_{f}, \tau_{r}, T_{f}$, and $T_{r}$ change dramatically at different positions. This could be partially due to contaminations from other components, such as potential further multiplicity, the possible large-scale outflow, and the possible high-velocity outflow associated with SMA-2. A more essential cause could be the oversimplified assumptions of the model itself, which assumes complete overlaps of the layers along the light of sight. A more detailed model should consider the two-dimensional geometry of the W3-SE region and include the influences of the core SMA2 , the additional $\mathrm{HCN}(3-2)$ blueshifted component, and other possible components. Although due to the limits of the model we can only analyze most of the fitted physical parameters qualitatively, an exception is the infall velocity $\left(V_{\text {in }}\right)$. It can be consistently fitted for the spectra from different positions; its value is always between 0.8 and $0.9 \mathrm{~km} \mathrm{~s}^{-1}$; and it is not sensitive to other fitted parameters. Actually it is also consistent with the velocity difference between the $\mathrm{HCN}(3-2)$ emission "bump" and the assumed systemic velocity.

Although fitting the $\mathrm{HCN}(3-2)$ spectrum toward SMA-1 is a tentative exercise, the fitting demonstrates a possible explanation: a complex including both high-velocity wings and an inverse P-Cygini profile for the observed $\mathrm{HCN}(3-2)$ line profile toward the SMA-1 region. A multiple-outflow model for the observed profile toward SMA-1 can be ruled out since no absorptions against the continuum are expected from the outflow gas with an excitation temperature of $\sim 75 \mathrm{~K}$, which is an order of magnitude larger than the brightness temperature of the continuum emission $(8 \mathrm{~K})$ at the observing wavelength. However, an alternative model with multiple outflows together with a cold envelope might fit the observed spectral profile. High-resolution observations of dense molecular lines at higher frequencies and a more sophisticated model are needed to unambiguously determine the nature of the observed profile toward SMA-1.

\subsection{The Nature of the Double Cores in W3-SE}

The W3-SE dust core is associated with a total mass of 65 $\pm 10 M_{\odot}$ in a $15^{\prime \prime}$ region, and is characterized by two continuum emission sources SMA-1 and SMA-2 with a projected angular separation 3".5 or 7000 AU. SMA-1 and SMA-2 contribute $1 / 2$ and $1 / 4$ of the total flux density in the $1.1 \mathrm{~mm}$ continuum, respectively (Zhu et al. 2010). Assuming a uniform distribution for both dust temperature and dust-to-gas ratio over the entire $\mathrm{W} 3-\mathrm{SE}$ region, a mass of $32 \pm 5 M_{\odot}$ is inferred for SMA-1 and $16 \pm 2 M_{\odot}$ for SMA-2. In the vicinity of the double protostellar cores, at least three additional mid-infrared sources have been revealed by Spitzer, indicating that the double cores located within or near SMA-1 and SMA2 might be a young cluster (Zhu et al. 2010). Both the SMA and CARMA observations of the molecular lines reveal a common filamentary envelope of molecular gas surrounding the two main protostellar cores, suggesting that SMA-1 and SMA-2 result from fragmentation of molecular gas in the same parent cloud.

The dust temperature of the W3-SE core region (covering SMA-1 and SMA-2) is $\sim 40 \mathrm{~K}$, indicating that the gas in the core region is likely to be heated by both internal and external energy sources. Indeed, a group of mid-IR sources are located east of SMA-1 and SMA-2, and mid-IR emission is also detected 
in SMA-1 (Zhu et al. 2010). The high temperature implies a relatively large Jeans mass. Assuming a mass of $65 M_{\odot}$ evenly distributed in a $15^{\prime \prime}$ region $(\sim 0.15 \mathrm{pc})$, a Jeans mass could be estimated as

$$
M_{\mathrm{J}}=1.2 \times 10^{5}\left(\frac{T}{100 \mathrm{~K}}\right)^{3 / 2}\left(\frac{\rho}{10^{-24} \mathrm{~g} \mathrm{~cm}^{-3}}\right)^{-1 / 2} \mu^{-3 / 2} M_{\odot}
$$

(Lang 1998), where $\mu$ is the mean molecular weight and assumed to be 2.35 . The inferred Jeans mass is $\sim 7 M_{\odot}$, far less than the derived masses of SMA-1 and SMA-2.

The observed infall signature might be due to global infall and/or inflow toward SMA-1. Although the spectra of $\mathrm{HCN}(3-2)$ and $\mathrm{HCO}^{+}(3-2)$ appear to be dominated by outflow, they still present significant redshifted absorption (see Figure 7). The $\mathrm{HCO}^{+}(1-0)$ spectrum toward the W3-SE core, which covers both SMA-1 and SMA-2, also shows the infall signature (Zhu et al. 2010). A global infall in W3-SE is likely to occur as the inferred Jeans mass is far less than the total mass of the W3-SE molecular core. However, both the spectral map (Figure 3) and the integrated intensity map (Figure 4(b)) show that the possible inverse P-Cygni profile in $\mathrm{HCN}(3-2)$ is present toward SMA-1, the major one of the double cores in W3-SE.

SMA-1 and SMA-2 may result from fragmentation of a common parent molecular cloud. The mechanisms for binary formation have been discussed by Tohline (2002). The location of SMA-1 and SMA-2 within a common filament (Figure 2(c)) suggests that the two cores result from a prompt fragmentation mechanism (Tohline 2002). Considering the projected separation, $d=7000 \mathrm{AU}\left(3^{\prime \prime} .5\right)$ for SMA-1 and SMA-2, and their total mass, $M_{\text {tot }} \approx 50 M_{\odot}$, they could be gravitationally bound if their actual separation is comparable to the projected one. Assuming SMA-1 and SMA-2 rotate around their mass center with a distance $d$, the orbital period $P=\left(2 \pi d^{3}\right)^{1 / 2}\left(G M_{\mathrm{tot}}\right)^{-1 / 2} \approx$ $3 \times 10^{4} \mathrm{yr}$ and the relative velocity of the two cores $\sim 2.5 \mathrm{~km} \mathrm{~s}^{-1}$. Although no significant difference in the radial velocities of the double cores is seen in our observations, this could be explained if the orbit is nearly face-on as implied by the large inclination angle $\left(\sim 70^{\circ}\right)$ of the high-velocity outflow in $\mathrm{HCN}(3-2)$ originating from SMA-1 (Section 4.1). The W3-SE protostellar system appears to be very young with an age $\sim 3 \times 10^{3} \mathrm{yr}$ inferred from the kinematic timescale of the outflow. This is an order of magnitude smaller than the orbital period if the molecular core W3-SE is to form a protobinary system, indicating it is still an unrelaxed system. However, the possibility that SMA-1 and SMA-2 will relax to a gravitationally bound binary system cannot be ruled out. Accretion could decrease the separation of the double cores and help the formation of a binary as suggested in the theory of dynamical hardening (Bonnell \& Bate 2005).

W3-SE can be compared with NGC 1333-IRAS 4, which was reported as a binary system consisting of two protostellar cores $4 \mathrm{~A}\left(9.2 M_{\odot}\right)$ and 4B $\left(4.0 M_{\odot}\right)$ with an angular separation $\sim 30^{\prime \prime}$ ( $\left.\sim 9000 \mathrm{AU}\right)$ at a distance of $\sim 300 \mathrm{pc}$ (Sandell et al. 1991). In follow-up observations with higher angular resolution, further multiplicities in both the sub-cores $4 \mathrm{~A}$ and $4 \mathrm{~B}$ have been revealed (e.g., Lay et al. 1995; Looney et al. 2000). Given the large masses for both SMA-1 and SMA-2 in W3-SE and the limited angular resolutions of the existing studies, SMA-1 and SMA-2 might turn out to be composed of multiple sub-cores in higher-resolution observations. The observed adjacent IR sources (Zhu et al. 2010) and multiple outflows also suggest further multiplicity in SMA-1 and SMA-2.

\section{SUMMARY}

Following our previous study with continuum and spectral lines at low angular resolution, we made high-resolution observations of multiple molecular lines toward the double protostellar cores SMA-1 and SMA-2 in W3-SE. The two protostellar cores are located in a molecular filamentary structure which shows an active star-forming region in W3-SE. A high-velocity bipolar outflow originating from SMA-1 is seen in $\mathrm{HCN}(3-2)$, with confirmation from other molecular lines, which shows a star formation activity in W3-SE. The morphology and the velocity profile of the bipolar outflow fit a bow-shock outflow model. SMA-1 and SMA-2 appear to result from fragmentation of a collapsing massive molecular core. Global collapse and gas infall are suggested by redshifted self-absorption observed in both $\mathrm{HCN}(3-2)$ and $\mathrm{HCO}^{+}(3-2)$. An inverse P-Cygni feature observed in the $\mathrm{HCN}(3-2)$ line toward the protostellar core SMA-1 suggests that SMA-1 is the major accretion source in W3-SE. The double protostellar cores in W3-SE are ideal targets for further observations with higher angular and spectral resolution at (sub-)millimeter wavelengths in order to understand the formation of clusters with intermediate-mass stars.

We are grateful to the staff of the SMA and CARMA telescopes who make these observations possible. We also thank the anonymous referee for his detailed comments and questions which have significantly improved the presentation of these results. We thank $\mathrm{Wu} \mathrm{Y}$. for the suggestions and discussions in forming the idea of this paper, selecting the research targets and providing the single-dish data of the targets, suggesting the suitable molecular probes, as well as the discussions in the analysis of the results. L.Z. is supported by the SAO pre-doctoral program during this research, and he is also supported by Grant 10873019 at NSFC when staying at Peking University.

\section{APPENDIX}

\section{BOW-SHOCK MODEL FOR OUTFLOW}

The bow-shock model (Downes \& Ray 1999) is one of the two popular models that provide mechanisms to accelerate molecular gas in an outflow. The observed features of the high-velocity bipolar outflow from SMA-1 appear to show the characteristics of a bow-shocked outflow as described in both numerical simulations and analytical models. In order to fit the $\mathrm{P}-\mathrm{V}$ diagram from the observed $\mathrm{HCN}(3-2)$ outflow with the bow-shock model, we have worked out the formulas to outline the boundary of a P-V diagram from a bow-shock surface based on the analytical model of Downes \& Ray (1999). In this model the bow-shock discontinuity is described as a rigid surface moving in the interstellar medium with a value of the bulk velocity $v$. For instance, in a cylindrical coordinate system, the locus of the bow shock in a blueshifted outflow lobe can be described as

$$
z=a-|r|^{s}, \quad s \geqslant 2,
$$

where $z$ is an axis along the direction of the bulk velocity of the bow-shock surface, $r$ is the radius perpendicular to axis $z$, and $s$ is a power-law index to describe the curvature of the bow-shock surface (see Figure 10). In addition, the bow-shocked surface is treated as a rigid body. We made the further assumptions used in the following reduction. In the bow-shock rest frame, the ambient medium moves toward the bow shock with a value of velocity $v$. As soon as the ambient molecular gas hits the surface of the bow shock, the motion of the ambient gas alters 
the direction that is tangential to the bow-shock surface with a value of velocity $v_{1}$ :

$$
v_{1}=\frac{v}{\sqrt{1+R^{2}}} \sqrt{\frac{1}{16}+R^{2}}
$$

where $R \equiv\left|\frac{v_{z}}{v_{r}}\right|=\left|\frac{d z}{d r}\right|=s(a-z)^{(s-1) / s}, \quad v_{z}$ and $v_{r}$ are the components of the velocity $v$ projected on the $z$ and $r$ directions (in the bow-shock fixed frame), and $a$ is the projected distance between bow-shocked tip and the origin of the outflow.

Considering the Doppler shift and the geometry described above, the complete surface of a bow-shocked bipolar outflow lobes can be divided into four separate parts, namely a combination of front and rear layers for each of the blueshifted and redshifted lobes. Each of the four boundaries corresponds to a curve in a quadrant of the $\mathrm{P}-\mathrm{V}$ space to outline a P-V diagram. Therefore, four separate functions $v_{B f}^{\prime}, v_{R f}^{\prime}, v_{B r}^{\prime}$, and $v_{R r}^{\prime}$ are used to describe the boundaries of bipolar outflow in the P-V diagram. Hereafter, the subscripts $B$ or $R$ represent blue or red velocities and $f$ or $r$ front or rear layers of a given bipolar outflow.

Given a front layer of a blueshifted bow shock, the observed radial velocity $v_{B f}^{\prime}$ in the LSR frame at a point of the bow-shock surface is a function of the tangential velocity $v_{1}$ :

$$
v_{B f}^{\prime}=\frac{v_{1}}{\sqrt{1+R_{B}^{2}}}\left(-\cos \alpha+R_{B} \sin \alpha\right)-v \sin \alpha+V_{\mathrm{sys}},
$$

where $V_{\text {sys }}$ is the systemic velocity of the source originating the bow-shock outflow, and $\alpha$ is the inclination angle, the angle between the axis of the outflow and the plane of the sky.

Therefore, substituting $v_{1}$ with Equation (A2), the observed radial velocity at a given point (Equation (A3)) can be rewritten as

$$
\begin{aligned}
& v_{B f}^{\prime}=\frac{v}{1+R_{B}^{2}}\left(-\cos \alpha+R_{B} \sin \alpha\right) \sqrt{\frac{1}{16}+R_{B}^{2}}-v \sin \alpha+V_{\mathrm{sys}}, \\
& v_{R f}^{\prime}=\frac{v}{1+R_{R}^{2}}\left(-\cos \alpha-R_{R} \sin \alpha\right) \sqrt{\frac{1}{16}+R_{R}^{2}}+v \sin \alpha+V_{\mathrm{sys}}, \\
& v_{B r}^{\prime}=\frac{v}{1+R_{B}^{2}}\left(\cos \alpha+R_{B} \sin \alpha\right) \sqrt{\frac{1}{16}+R_{B}^{2}}-v \sin \alpha+V_{\mathrm{sys}}, \\
& v_{R r}^{\prime}=\frac{v}{1+R_{R}^{2}}\left(\cos \alpha-R_{R} \sin \alpha\right) \sqrt{\frac{1}{16}+R_{R}^{2}}+v \sin \alpha+V_{\mathrm{sys}},
\end{aligned}
$$

where $R_{B}=s_{B}\left(a_{B}-z\right)^{\left(s_{B}-1\right) / s_{B}}$ and $R_{R}=s_{R}\left(a_{R}+z\right)^{\left(s_{R}-1\right) / s_{R}}$. The values of the bow-shock velocities for the blueshifted and redshifted outflow lobes have been assumed to be the same in Equation (A4). This assumption is valid since the line widths of the blueshifted and redshifted wings are very similar as discussed in Section 3.1. We can further simplify the model for the velocity profiles by assuming $s_{R}=s_{B}=2$. In addition, the projected distances between the bow-shock tips and the origin of the outflow $\left(a_{B}\right.$ and $\left.a_{R}\right)$ can be determined directly from the outflow image. The systemic velocity $V_{\text {sys }}$ can be determined from the peak velocity of hot molecular lines. For SMA-1, $V_{\text {sys }}=-39.1 \mathrm{~km} \mathrm{~s}^{-1}$ is used (see Section 3.4). Thus, the remaining free parameters in Equation (A4) are the inclination angle $\alpha$ and the bow-shock velocity $v$.
Equation (A4) describes the observed radial velocities along the boundaries of a bow-shock outflow as a function of $z$. Given a cylindrical coordinate system with an origin that is the same as that of the outflow (as illustrated in Figure 10), the position on the sky plane $\left(r^{\prime}, z^{\prime}\right)$ can be transferred from the coordinates $(r, z)$ on the bow-shock surface for a given inclination angle $\alpha$ :

$$
\begin{aligned}
& z^{\prime}=z \cos \alpha+r \sin \alpha \\
& r^{\prime}=-z \sin \alpha+r \cos \alpha
\end{aligned}
$$

Using Equations (A1) and (A5), the sky position $z^{\prime}$ can be related to the outflow coordinate $z$ for each of the four boundaries:

$$
\begin{aligned}
& z_{B f}^{\prime}=z \cos \alpha-\left(a_{B}-z\right)^{1 / 2} \sin \alpha \\
& z_{R f}^{\prime}=z \cos \alpha-\left(a_{R}+z\right)^{1 / 2} \sin \alpha \\
& z_{B r}^{\prime}=z \cos \alpha+\left(a_{B}-z\right)^{1 / 2} \sin \alpha \\
& z_{R r}^{\prime}=z \cos \alpha+\left(a_{R}+z\right)^{1 / 2} \sin \alpha .
\end{aligned}
$$

Thus, the observed radial velocities (Equation (A4)) for a given bow-shocked bipolar outflow can be implicitly related to the coordinate $z^{\prime}$ on the sky plane with Equation (A6) by eliminating the argument $z$. With the four implicit functions $v^{\prime}=v^{\prime}\left(z^{\prime}\right)$, we can outline a P-V diagram along the projected major axis of a bipolar outflow using the bow-shocked model. Note that in the above deduction we worked out the geometrical relation without including optical depth effect.

\section{REFERENCES}

Attard, M., Houde, M., Novak, G., et al. 2009, ApJ, 702, 1584

Blitz, L., Fich, M., \& Stark, A. A. 1982, ApJS, 49, 183

Bonnell, I. A., \& Bate, M. R. 2005, MNRAS, 362, 915

Di Francesco, J., Myers, P. C., Wilner, D., Ohashi, N., \& Mardones, D. 2001, ApJ, 562, 770

Downes, T. P., \& Ray, T. P. 1999, A\&A, 345, 977

Jorgensen, J. K., Hogerheijde, M. R., van Dishoeck, E. F., Blake, G. A., \& Schöier, F. L. 2004a, A\&A, 413, 993

Jorgensen, J. K., Schöier, F. L., \& van Dishoeck, E. F. 2004b, A\&A, 416, 603

Lang, K. R. 1998, Astrophysical Formulae Vol. I (3rd ed.; Berlin: Springer)

Lay, O. P., Carlstrom, J. E., \& Hills, R. E. 1995, ApJ, 452, L73

Lee, C. F., Mundy, L. G., Reipurth, B., Ostriker, E. C., \& Stone, J. M. 2000, ApJ, 542,925

Lee, C. F., Stone, J. M., Ostriker, E. C., \& Mundy, L. G. 2001, ApJ, 557, 429

Looney, L. W., Mundy, L. G., \& Welch, W. J. 2000, ApJ, 529, 477

McKee, C. F., \& Ostriker, E. C. 2007, ARA\&A, 45, 565

Myers, P. C., Mardones, D., Tafalla, M., Williams, J. P., \& Wilner, D. J. 1996, ApJ, 465, L133

Peretto, N., André, P., \& Belloche, A. 2006, A\&A, 445, 979

Ruch, G. T., Jones, T. J., Woodward, C. E., Polomski, E. F., \& Gehrz, R. D. 2007, ApJ, 654, 338

Sandell, G., Aspin, C., Duncan, W. D., Russell, A. P. G., \& Robson, E. I. 1991, ApJ, 376, L17

Sault, R. J., Teuben, P. J., \& Wright, M. C. H. 1995, in ASP Conf. Ser. 77, Astronomical Data Analysis Software and Systems IV, ed. R. A. Shaw, H. E. Payne, \& J. J. E. Hayes (San Francisco, CA: ASP), 433

Tohline, J. E. 2002, ARA\&A, 40, 349

Ward-Thompson, D., \& Buckley, H. D. 2001, MNRAS, 327, 955

Ward-Thompson, D., Buckley, H. D., Greaves, J. S., Holland, W. S., \& André, P. 1996, MNRAS, 281, L53

Wilson, T. L., Rohlfs, K., \& Hüttemeister, S. 2009, Tools of Radio Astronomy (5th ed.; Berlin: Springer)

Wu, Y., Henkel, C., Xue, R., Guan, X., \& Miller, M. 2007, ApJ, 669, L37

Xu, Y., Reid, M. J., Zheng, W. W., \& Menten, K. M. 2006, Science, 311, 54

Zhu, L., Wright, M., Zhao, J.-H., \& Wu., Y. 2010, ApJ, 712, 674 